\title{
The dependence of winter aurora on interplanetary parameters
}

\author{
J. B. Baker, ${ }^{1}$ A. J. Ridley, V. O. Papitashvili, and C. R. Clauer \\ Space Physics Research Laboratory, University of Michigan, Ann Arbor, Michigan, USA
}

Received 26 February 2002; revised 30 September 2002; accepted 4 October 2002; published 22 February 2003.

[1] The dependence of the northern winter aurora on interplanetary parameters is examined for images obtained from the Polar spacecraft Ultraviolet Imager (UVI) during the month of January 1997. Linear correlation analysis is used to relate auroral brightness to interplanetary parameters as a function of magnetic latitude (MLAT) and magnetic local time (MLT). Spatial maps of maximum magnitude correlation coefficient and optimal lag times are presented. In addition, the Method of Natural Orthogonal Components (MNOC) is applied to UVI images and an orthogonal set of eigenmodes of auroral behavior is produced. The dominant eigenmodes are determined to be (1) the main auroral oval brightness, (2) polar cap contraction/expansion, (3) dawn/dusk aurora, and (4) midnight sector brightening/ quenching. The temporal coefficients for the various eigenmodes are correlated separately with solar wind parameters and interplanetary magnetic field (IMF) components. The dominant influence of the negative $B_{z}$ component is confirmed, but the timescale of its influence is found to vary widely. The MNOC procedure allows some of the more subtle relationships between the aurora and the interplanetary parameters to be isolated, particularly those associated with the solar wind density and IMF $B_{x}$ and $B_{y} . \quad$ INDEXTERMS: 2704 Magnetospheric Physics: Auroral phenomena (2407); 2784 Magnetospheric Physics: Solar wind/ magnetosphere interactions; 2736 Magnetospheric Physics: Magnetosphere/ionosphere interactions; 2134 Interplanetary Physics: Interplanetary magnetic fields; KEYWORDS: aurora, UVI, IMF, eigenmode, eigenvalue

Citation: Baker, J. B., A. J. Ridley, V. O. Papitashvili, and C. R. Clauer, The dependence of winter aurora on interplanetary parameters, J. Geophys. Res., 108(A4), 8009, doi:10.1029/2002JA009352, 2003.

\section{Introduction}

[2] The interplanetary medium is an important source of mass and energy for the magnetosphere and has a direct influence on the generation of auroral precipitation at high latitudes. The solar wind-magnetosphere interaction is thought to be a combination of magnetic reconnection and viscous interactions at the magnetopause boundary. Magnetic reconnection is the dominant coupling mechanism when the interplanetary magnetic field (IMF) is directed southward. Viscous interactions include the Kelvin-Helmholtz instability during high solar wind velocity and impulsive plasma penetrations during sudden changes in dynamic pressure. The net power transferred to the magnetosphere from the interplanetary medium is generally thought to be of the order of $10^{6} \mathrm{MW}$, of which, viscous interactions probably account for perhaps 10-20\% [e.g., Reiff and Luhmann, 1986; Papitashvili et al., 1994]. It is thus natural to correlate magnetospheric behavior with conditions measured in the solar wind and IMF. A number of solar windmagnetosphere coupling functions have been postulated for the dependence of energy input to the magnetosphere on interplanetary parameters [e.g., Rostoker et al., 1972; Mae-

\footnotetext{
${ }^{1}$ Now at Applied Physics Laboratory, Johns Hopkins University, Laurel MD, USA.

Copyright 2003 by the American Geophysical Union. 0148-0227/03/2002JA009352
}

zawa, 1979; Murayama, 1982; Baker, 1986; Liou et al., 1998].

[3] Much has been learned over the years by relating steady and transient features of the global aurora with ionospheric convection, field aligned currents, particle precipitation and IMF orientation [e.g., Murphree and Elphinstone, 1987; Jankowska et al., 1990; Murphree et al., 1989; Zanetti et al., 1990; Elphinstone et al., 1990; Elphinstone and Hearn, 1993; Vo and Murphree, 1995; Sitar et al., 1998]. Lassen and Danielson [1978] used all-sky camera images to show that during southward IMF the occurrence rate of auroral oval arcs is higher and the size of the auroral oval is expanded. Meng [1979] observed that the auroral oval contracts during northward IMF and that the $B_{y}$ component has some control over the dawn-dusk asymmetry of this contraction: for positive (negative) $B_{y}$ the contraction occurs in the evening (morning) sector and the center of the northern polar cap is displaced dawnward (duskward). The $B_{y}$ component has also been observed to control dawn-dusk asymmetries in ionospheric convection and the behavior of auroral arcs during northward IMF [Cowley, 1981a; Murphree et al., 1982]. Likewise, it has been suggested that the $B_{x}$ component might be responsible for similar asymmetries and interhemispherical effects [Cowley, 1981b; Crooker et al., 1985].

[4] Satellite-borne auroral imagers provide a unique global perspective with which to examine the aurora. They provide good resolution within a single data set, allowing easy separation of temporal and spatial features. Recent 
studies have used auroral images from the Polar spacecraft Ultraviolet Imager (UVI) to investigate the IMF dependence of the aurora. Global auroral power during summer months has been found to be larger by $15-20 \%$ for $B_{y}<0$ than for $B_{y}>0$ [Liou et al., 1998]. This effect has been attributed to a partial penetration of the IMF $B_{y}$ into the near-Earth plasma sheet giving rise to interhemispheric currents and an associated Northern Hemisphere aurora [Lui, 1984; Wing et al., 1995]. Shue et al. [2001] produced average patterns of UVI-measured aurora binned by IMF clock angle and season and showed that the afternoon auroral bright spot during large northward IMF or small $B_{z}$ is more distinct from the nightside aurora for $B_{y}<0$ than for $B_{y}>0$.

[5] The Method of Natural Orthogonal Components (MNOC) is a statistical technique that calculates a "natural" set of orthogonal basis eigenvectors (or eigenmodes) for a data set [Kendall and Stuart, 1976]. The MNOC procedure uses the data to produce the basis, in contrast with spherical harmonic or Fourier methods that use predetermined basis vectors. The MNOC procedure solves an eigenvalue problem to determine the basis vectors from an autocorrelation matrix of the data. Previous geophysical applications of the MNOC technique include the separation of the geomagnetic field into long and short wavelength components [Frynberg, 1975; Pushkov et al., 1976; Rotanova et al., 1982], and the development of an improved method for calculation of magnetic K indices [Golovkov et al., 1978; Papitashvili et al., 1992]. More recently, Sun et al. [1998] applied the MNOC procedure to ionospheric equivalent current distributions determined from magnetogram inversion. They found that the first two eigenmodes, on average, accounted for $83 \%$ of the total current over a 3 day period and each could be identified respectively with the driven and unloading components of ionospheric activity.

[6] In this paper, two statistical methods are used to investigate the influence that interplanetary parameters have on the global auroral distribution measured by UVI. First, linear correlation analysis is used to relate auroral brightness with interplanetary parameters as a function of magnetic latitude (MLAT) and magnetic local time (MLT). Spatial maps of maximum magnitude correlation coefficient and optimal lag times are presented and discussed (section 4). Second, the MNOC procedure is applied to UVI images to determine the dominant eigenmodes of auroral activity (section 5). It is demonstrated that the first few eigenmodes can be interpreted in terms of common auroral processes and morphologies. Detailed analysis of the temporal coefficients for each eigenmode gives an indication of the timescales on which the aurora changes in response to forcing by the solar wind and IMF. The paper concludes with a discussion of the results in section 6 and a summary in section 7 .

\section{Data Preparation}

[7] In this section, the various data sets are described. These include auroral images obtained from the Polar spacecraft UVI and measurements of interplanetary parameters obtained by instrumentation aboard the Wind spacecraft located in the interplanetary medium. The method for propagating the interplanetary parameters to the Earth is also described.

\subsection{Polar UVI}

[8] The UVI aboard the Polar spacecraft is a reflective CCD camera that produces images of the aurora in the far ultraviolet $(130-190 \mathrm{~nm})$ region of the electromagnetic spectrum [see Torr et al., 1995 for details]. The system is mounted on a single axis despun platform that provides inertial viewing for extended periods of time. The instrument images onto an intensified CCD detector array comprising 228 by 200 pixels. The angular resolution $\left(0.036^{\circ}\right)$ translates to a spatial resolution of $40 \mathrm{~km}$ when viewed from apogee. A filter wheel selects from five available filters: OI 1304, OI 1356, N2 LBHs, N2 LBH1, and long wavelength scattered sunlight.

[9] All UVI images used in this paper were taken using the LBHl (140-160 nm) filter and have an integration time of $37 \mathrm{~s}$. The auroral luminosity observed with the LBHl filter is directly proportional to the energy flux of precipitating electrons [Germany et al., 1997]. An emission altitude of $120 \mathrm{~km}$ has been assumed when performing coordinate transformations. The images were taken during winter so removal of day glow was not necessary. However, they have been flat field corrected and had a uniform background removed. The images have also been partially corrected for the Polar spacecraft platform wobble. The orientation of the wobble during the period of study was aligned approximately parallel to the noon-midnight meridian. At its worst, the platform wobble could be responsible for errors as large as $2.5^{\circ}$ of MLAT if not removed. The dewobble algorithm works by searching for pairs of pixels along the wobble direction that are of comparable brightness and separated by a distance equal to the known amplitude of the wobble. Such pixels are convolved together at their midpoint.

\subsection{Wind Magnetic Fields Investigation (MFI) and Solar Wind Experiment (SWE)}

[10] Measurements of the solar wind plasma and IMF used in this paper were obtained by the SWE and MFI aboard the Wind spacecraft. MFI consists of two triaxial fluxgate magnetometers mounted on a $12 \mathrm{~m}$ deployable boom [Lepping et al., 1995]. SWE is a six axis ion-electron spectrometer that provides three-dimensional velocity distribution functions for solar wind ions and electrons [Ogilvie et al., 1995]. MFI and SWE data have been despiked and propagated to the magnetosphere using ballistic propagation. The instantaneous X-distance and solar wind speed for each data record were used to calculate a transit time to the bow shock. An additional 10 min was added to account for propagation through the magnetosheath and into the inner magnetosphere. All data records were shifted by this variable transit time, averaged, and then gridded at a resolution of $1 \mathrm{~min}$. A running average filter with a window of $15 \mathrm{~min}$ has been used to remove spikes and high frequency variations.

\section{Study Period: January 1997}

[11] The data set of UVI images spans the full month of January 1997 and comprises 10,576 images taken with the LBHl filter and having an integration time of $37 \mathrm{~s}$. All UVI images have been transformed into Apex magnetic coordinates [Richmond, 1995] and averaged onto a spatial grid 
measuring 1 hour MLT by $1^{\circ}$ MLAT. Figure 1 shows the spatial coverage of UVI images on this grid during the entire month. The gray-scale contouring shows the number of UVI images that contributed to each bin on the MLTMLAT spatial grid. Magnetic noon is directed toward the top. A lowest latitude of $60^{\circ}$ was selected to bin the data. This was chosen somewhat arbitrarily but also guided by the fact that the MNOC results in section 5 suggest that this is a natural boundary for normal auroral activity.

[12] Figure 2 shows MFI measurements of the IMF $B_{x}$, $B_{y}$, and $B_{z}$ components and SWE measurements of the solar wind speed and density during January 1997 (upper five plots). A prominent feature is the magnetic cloud event of 10 and 11 January [see Spann et al., 1998]. More generally, it can be seen that there is a significant range of values for the solar wind and IMF parameters during the course of the month. A 1 min proxy AE index is also provided second from the bottom. It should be noted that this is not the official AE index provided through the World Data Center (WDC). It has been calculated using the same methodology used to generate the WDC AE index but used data from all available magnetometers at auroral latitudes (in this case 57), rather than just the $10-13$ official AE stations. The bottom plot shows the maximum auroral photon flux (photons $/ \mathrm{cm}^{2} / \mathrm{s}$ ) obtained from the binned UVI images. The cadence of this time series is approximately 3-5 min when UVI imaging is available in the Northern Hemisphere, depending on the instantaneous UVI filter cycle. This quantity is well correlated with the $\mathrm{AE}$ index when the spatial coverage of UVI covers the nightside $(C=0.81)$. This is to be expected because both quantities are measures of the maximum geophysical disturbance measured over the polar region.

[13] It is possible that the interplanetary parameters may have intercorrelations that might affect the interpretation of later results. It is therefore useful to examine the intercorrelations first and this is done in Table 1. The upper triangle is for $B_{z}<0$, the lower triangle for $B_{z}>0$. In general, the interplanetary parameters were more strongly correlated during northward IMF than they were during southward IMF. Also noteworthy is the fact that the dynamic pressure, $P$, has a higher correlation with the solar wind density, $N$, than it has with the speed, $V_{x}$. It should be emphasized that the relationships exhibited in Table 1 apply for the limited period during January 1997 and do not necessarily reflect the general properties of the interplanetary medium.x

\section{Linear Correlation Analysis}

[14] In this section, time-lagged correlation analysis is used to determine the spatial regions in which the aurora shows a linear correlation with the IMF and solar wind, and the relevant timescales over which the correlations apply. The interplanetary data were restricted to isolate average behavior from some of the more extreme conditions exhibited during January 1997. In particular, intervals for which IMF $B_{z}$ and/or total IMF magnitude were greater than 8 nT were excluded. In addition, the solar wind velocity and density were respectively restricted to be in the ranges $300-$ $600 \mathrm{~km} / \mathrm{s}$ and $5-12$ particles $/ \mathrm{cm}^{3}$. These restrictions are shown in Table 2. The general approach is to correlate interplanetary parameters with UVI auroral brightness at

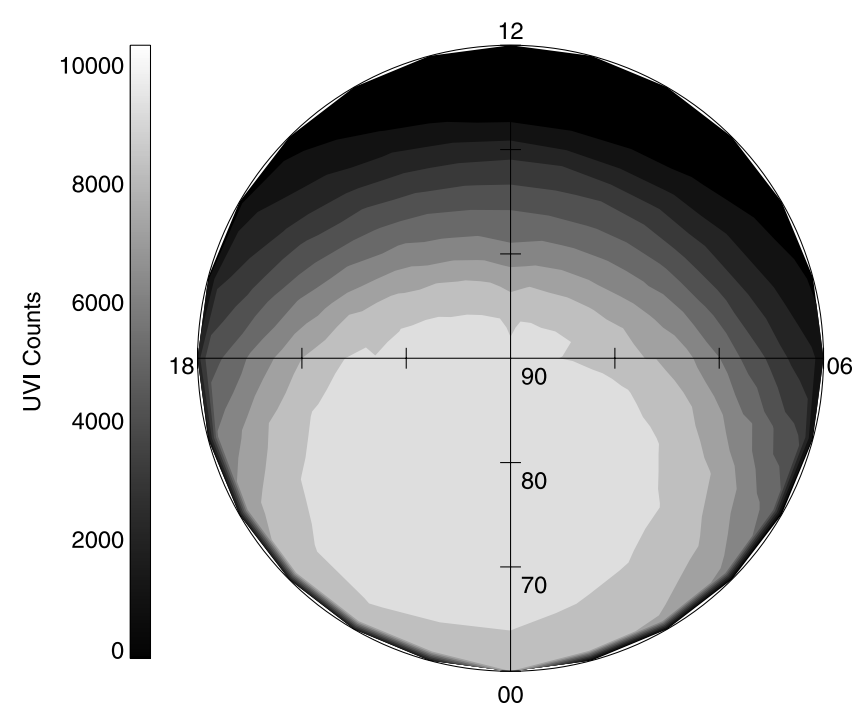

Figure 1. Spatial coverage of UVI images during January 1997. The plot is rendered in Apex MLAT-MLT coordinates down to a lowest latitude of $60^{\circ}$ with magnetic noon toward the top.

each point on the MLAT-MLT grid using a variable time lag up to a maximum of 3 hours. The maximum magnitude correlation (positive or negative) and corresponding optimal lag time are then contoured as a function of MLAT and MLT.

[15] These correlation results can be used to make inferences about the physical mechanisms responsible for generation of the aurora. The assumption is made that each correlation can be related to an important solar windmagnetosphere coupling process that energizes magnetospheric plasma and produces auroral precipitation. Three factors are important when interpreting the correlation results in this way: (1) the MLAT-MLT location, (2) the optimal timescale, and (3) the interplanetary parameter under consideration. The MLAT-MLT location of the correlation gives an indication of the magnetospheric source region of the precipitation and provides clues about the nature of the energization mechanism. For example, midnight sector aurora is generally produced by precipitation from the midtail energized by magnetic reconnection, while dusk or dawn sector aurora is more likely associated with interactions at the flanks of the magnetosphere and may be viscous in nature. The timescale of the correlation indicates whether the energy associated with a particular interplanetary influence is released to the ionosphere relatively quickly (directly driven activity), or is instead stored for some intermediate period of time for later release (loadingunloading activity). Ordinarily, one might expect viscous interactions to operate on short timescales and magnetic reconnection to operate over longer loading-unloading timescales.

\subsection{Results}

[16] Figures 3 and 4 show correlation maps during southward and northward IMF respectively. In each figure, the first and third columns show the maximum magnitude correlation coefficient, while the third and fourth columns show the appropriate timescales for these correlations. Each 

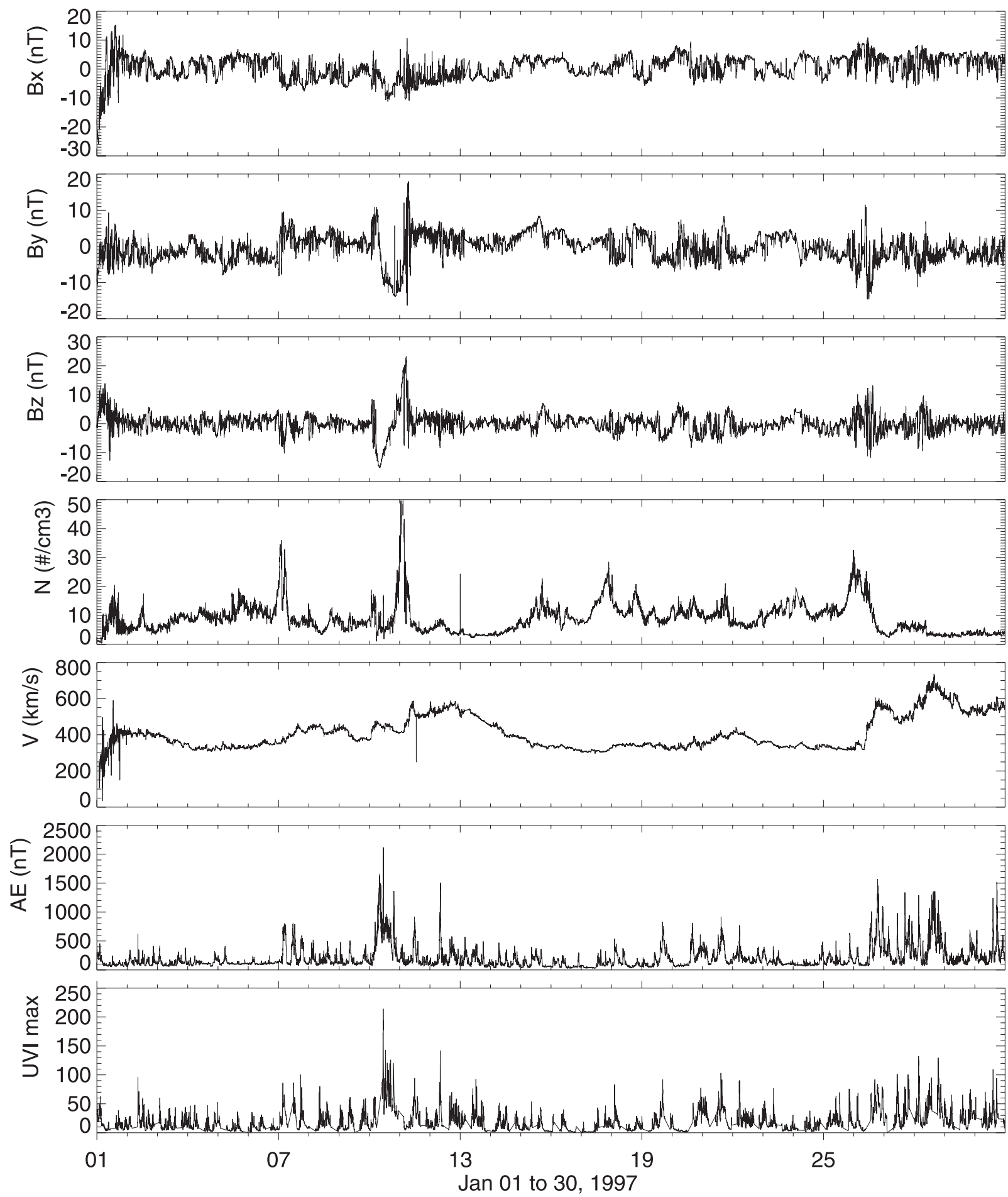

Figure 2. Interplanetary and magnetospheric parameters during January 1997. From top to bottom: (a) IMF $B_{x}$, (b) IMF $B_{y}$, (c) IMF $B_{z}$, (d) solar wind density, (e) solar wind speed, (f) AE index, and (g) UVI maximum binned photon flux (photons $/ \mathrm{cm}^{2} / \mathrm{s}$ ).

contour plot is rendered in the same Apex MLAT-MLT coordinates used in Figure 1 and the lowest latitude shown is $60^{\circ}$. The color bars on the left and right respectively represent the maximum magnitude correlation coefficient (blue-red) and corresponding optimal lag time (red-black). Regions of positive (negative) correlation are colored red (blue); short (long) timescales are colored red-pink (blue- black). Each pair of contour maps are labeled with the appropriate interplanetary parameter under consideration. On the left (first two columns) are IMF $B_{z}$, solar wind $N, V_{x}$, and $P$. On the right are positive and negative values of IMF $B_{x}$ and $B_{y}$. For consistency it is the strength of the IMF component, whether positive or negative, that has been correlated against UVI brightness. It should be noted that 
Table 1. Linear Correlation Coefficients for Solar Wind and IMF Parameters $^{\mathrm{a}}$

\begin{tabular}{ccccccc}
\hline & $B_{x}$ & $B_{y}$ & $B_{z}$ & $V_{x}$ & $N$ & $P$ \\
\hline$B_{x}$ & & -0.19 & 0.08 & -0.10 & -0.01 & -0.10 \\
$B_{y}$ & -0.38 & & 0.05 & 0.03 & 0.10 & 0.10 \\
$B_{z}$ & -0.09 & -0.01 & & -0.01 & -0.16 & -0.22 \\
$V_{x}$ & -0.20 & 0.06 & -0.04 & & -0.59 & -0.02 \\
$N$ & 0.00 & -0.08 & 0.41 & -0.40 & & 0.76 \\
$P$ & -0.07 & -0.11 & 0.46 & 0.01 & 0.88 & \\
\hline
\end{tabular}

${ }^{\mathrm{a}}$ Upper (lower) triangle is for southward (northward) IMF.

the time lag maps have been median filtered with a width of 3 grid cells. This was done because the time dependence of the correlations were very weak in some cases, making the identification of a precise optimal time lag difficult, and producing noisy patterns. The aim of this analysis is to extract macroscopic features from the maps and so it was decided to median filter the time lag maps. This was not necessary for the correlation maps.

\subsubsection{Southward IMF}

[17] Figure 3 clearly identifies the spatial regions in which the aurora has a strong linear relationship with the interplanetary parameters during southward IMF. Most notably, negative $B_{z}$ is highly correlated with auroral brightness throughout most of the auroral oval region on timescales from 50 to $150 \mathrm{~min}$. The longest timescale correlations are associated with the low and high latitude portions of the nightside auroral oval where the aurora becomes active during substorms. The weakest correlations are found in the premidnight region, presumably because of the episodic auroral activity that occurs in this sector during substorms. It is particularly interesting to note that the dayside auroral zone shows a negative correlation with negative $B_{z}$ on short timescales. This can be attributed to a fading of aurora at the latitude of the quiet time dayside auroral oval when magnetic reconnection at the magnetopause produces an expansion of the auroral oval to lower latitudes.

[18] Figure 3 also shows some interesting asymmetries in the response of the aurora to positive and negative values of the IMF $B_{x}$ and $B_{y}$ components during southward IMF. Generally, auroral brightness has a much stronger correlation with negative $B_{x}$ and $B_{y}$ than it does with positive $B_{x}$ and $B_{y}$. In some regions, the correlations with positive $B_{x}$ and $B_{y}$ are actually negative. The strongest correlations with negative $B_{y}$ are found in the early morning sector where auroral activity is less affected by substorms. On the dayside, there are negative correlations with IMF $B_{y}$, similar to that found for negative $B_{z}$, which are probably associated once again with motion of the dayside auroral oval. The negative correlation is higher and more widespread for positive $B_{y}$ than for negative $B_{y}$. This is consistent with the study of Shue et al. [2001], who found that the afternoon sector auroral oval shifts to higher latitudes for $B_{y}>0$ under southward IMF conditions. Likewise, the asymmetric dependence on IMF $B_{x}$ can be explained by a favorable antiparallel reconnection geometry at the Northern Hemisphere magnetopause during negative $B_{x}$ driving a stronger aurora, in accordance with the study of Cowley [1981b].

[19] Turning now to the solar wind parameters in Figure 3 , it can be seen that the strongest and most widespread correlations are associated with $V_{x}$. The timescales of these correlations are $0-60 \mathrm{~min}$ in the main auroral zone and much longer at high latitudes. The wide variety of timescales indicates that $V_{x}$ may be important in the generation of both directly driven and loading-unloading auroral activity. This result is not unexpected because the solar wind velocity is known to control both the strength of the viscous drag on the magnetosphere by the solar wind, as well as the rate at which magnetic flux can be transported to the subsolar point for reconnection with the geomagnetic field. By contrast, the dependence of the aurora on the dynamic pressure is generally weaker than for $V_{x}$ and acts over much longer timescales, particularly in the morning sector. This suggests that the dynamic pressure is generally less important than $V_{x}$ in solar wind-magnetosphere coupling. The correlation with the density $N$ is generally negative and acts over short to moderate timescales where negative, and very long timescales where positive. It should be cautioned that the dependence on $N$ is probably contaminated by the strong negative intercorrelation between $N$ and $V_{x}$ during the study interval (see Table 1).

\subsubsection{Northward IMF}

[20] The correlation results in Figure 4 during northward IMF are generally stronger on the dayside than on the nightside. A sharp decline in the influence of the $B_{z}$ component when the IMF turns northward is particularly evident. There is essentially no correlation between positive $B_{z}$ and the brightness in the main auroral zone. Instead, there is a relatively high correlation in two localized regions located in the dawn and dusk sectors centered at $80^{\circ}-85^{\circ}$ latitude. These correlations are on short timescales and are probably associated with polar cap arc activity. Also of interest is the fact that the dynamic pressure shows moderate correlation on short timescales across the dayside from dawn to dusk, but is anticorrelated with the aurora in the midnight region. The relative influence of the solar wind velocity also seems to be greater during northward IMF, particularly on the dawn side, and the optimal timescale of the correlations tend to be shorter. All of these observations are consistent with a decrease in unloading auroral activity from the magnetotail and a relative increase in directly driven viscous activity at the magnetopause during northward IMF.

\section{Method of Natural Orthogonal Components}

[21] In this section, the MNOC is described and applied to UVI images during January 1997. The description of the procedure is reproduced from the study of Sun et al. [1998]. The interested reader is encouraged to refer to the text by Kendall and Stuart [1976] for a more detailed treatment.

\subsection{Procedure}

[22] We suppose that a data set, $E_{i, j}$, is a function of time $t_{i}$ $(i=1,2, \ldots m)$ and location $r_{j}(j=1,2, \ldots n)$. We seek an expansion of the data in terms of a set of orthogonal eigenmodes $X_{j}^{k}$, and their corresponding temporal coeffi-

Table 2. Restrictions Applied to the Interplanetary Data

\begin{tabular}{lcccc}
\hline & $|B|(\mathrm{nT})$ & $\left|B_{z}\right|(\mathrm{nT})$ & $V_{x}(\mathrm{~km} / \mathrm{s})$ & $N\left(/ \mathrm{cm}^{3}\right)$ \\
\hline $\operatorname{Min}$ & 0 & 0 & 300 & 5 \\
$\operatorname{Max}$ & 8 & 8 & 600 & 12 \\
\hline
\end{tabular}




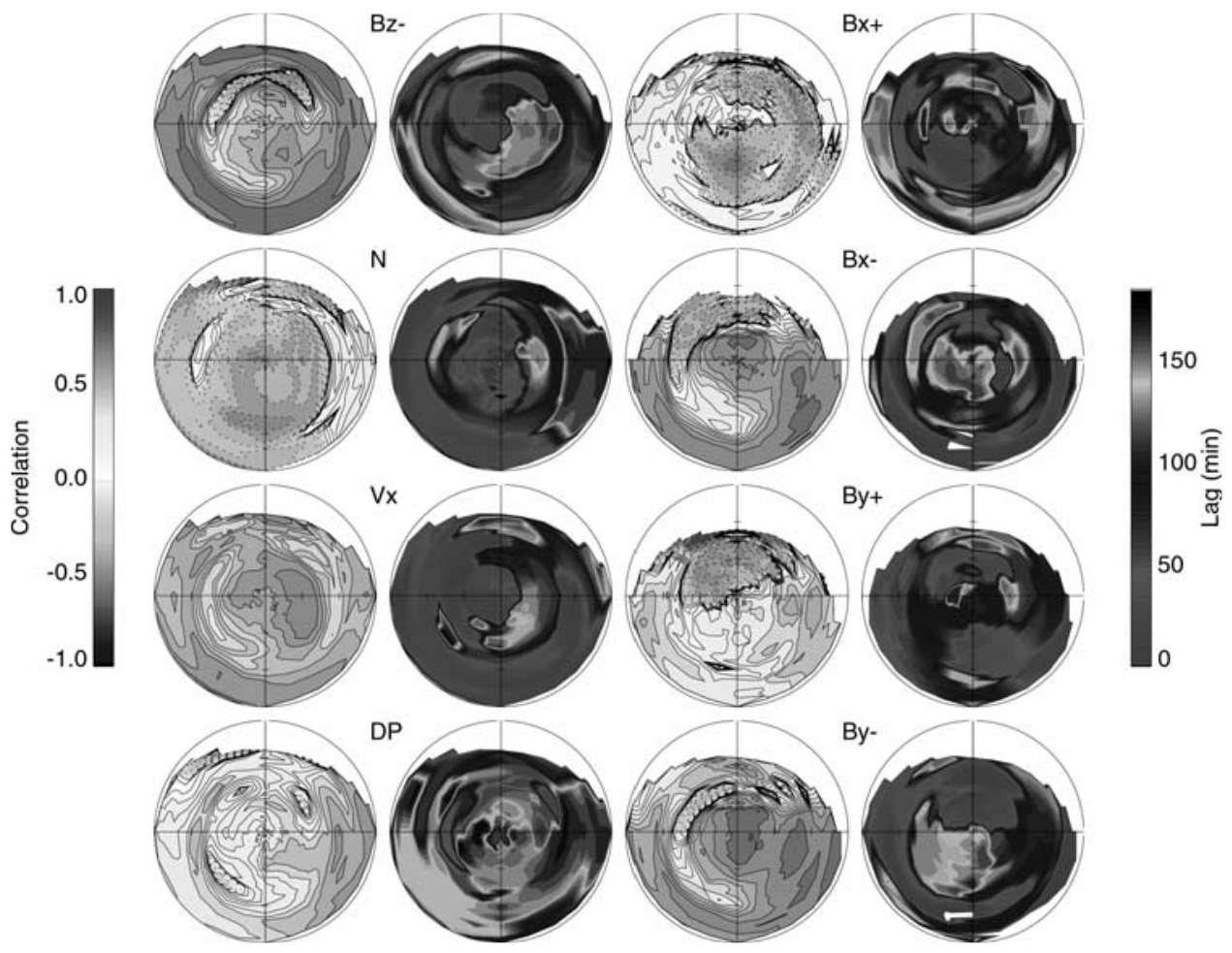

Figure 3. Contours of maximum magnitude correlation (columns 1 and 3; left color scale) and optimal lag time (columns 2 and 4; right color scale) between interplanetary parameters and UVI-measured aurora during southward IMF. Representation is in Apex MLAT-MLT coordinates down to a lowest latitude of $60^{\circ}$ with magnetic noon at the top. See color version of this figure at back of this issue.

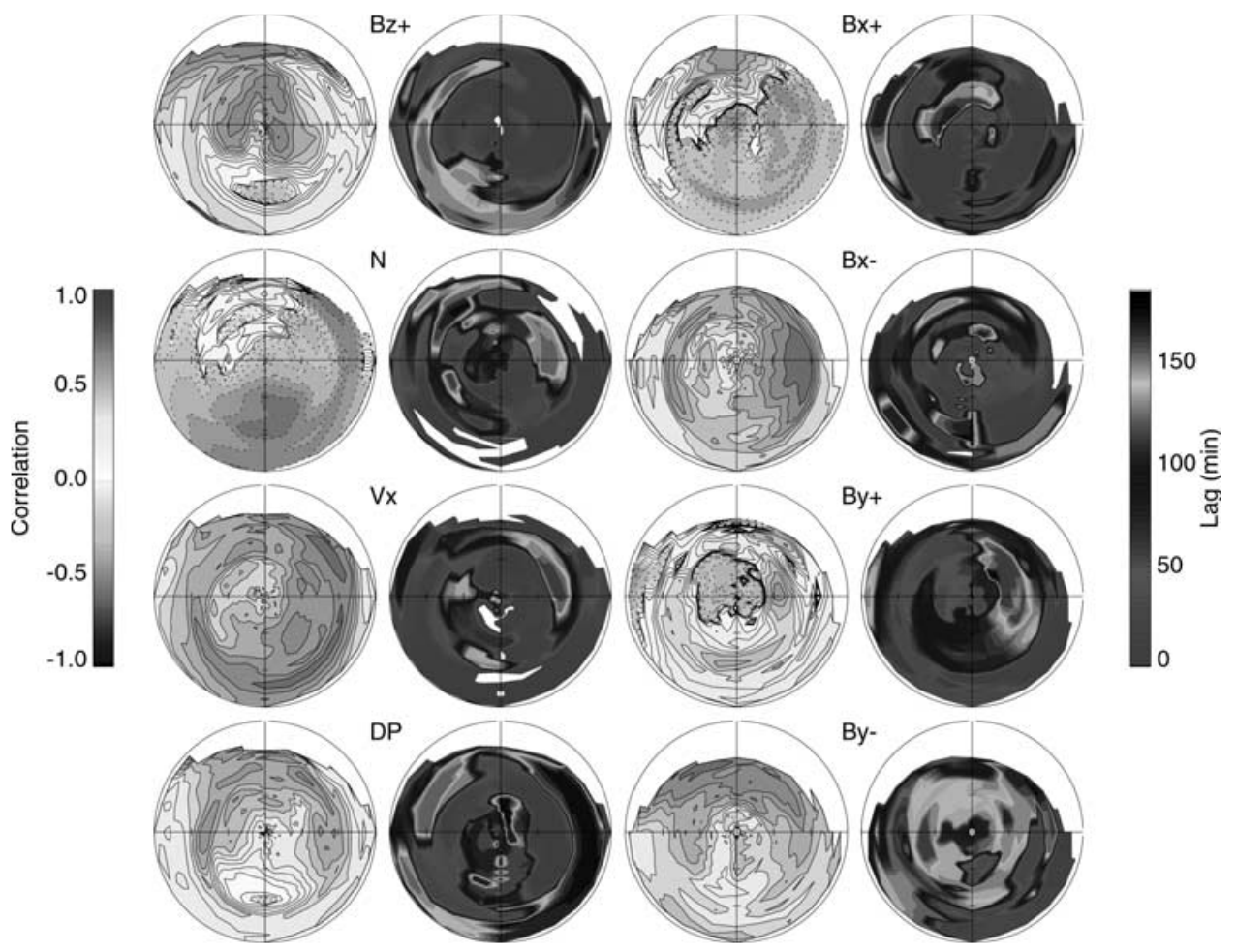

Figure 4. Contours of maximum magnitude correlation and optimal lag time between interplanetary parameters and UVI-measured aurora during northward IMF (in same format as Figure 3). See color version of this figure at back of this issue. 
cients $T_{i}^{k}$, for orders $k=1,2, \ldots h \leq n$. Such an expansion can be represented as,

$$
E_{i, j}=\sum_{k=1}^{h} T_{i}^{k} X_{j}^{k}
$$

where the indices $i, j$, and $k$, respectively, represent time, space, and order of the eigenmode expansion. The orthogonality condition is given by,

$$
\sum_{j=1}^{n} X_{j}^{l} X_{j}^{p}=0 \quad l \neq p .
$$

[23] To solve for the eigenmodes $X_{j}^{k}$ and the temporal coefficients $T_{i}^{k}$ it is necessary to minimize the following quantity with respect to $X_{j}^{k}$ and $T_{i}^{k}$ :

$$
\delta_{n}=\sum_{i=1}^{m} \sum_{j=1}^{n}\left[E_{i j}-\sum_{k=1}^{h} T_{i}^{k} T_{j}^{k}\right]^{2} .
$$

This yields the following equation for the temporal coefficients:

$$
T_{i}^{k}=\frac{\sum_{j=1}^{n} E_{i j} X_{j}^{k}}{\sum_{j=1}^{n}\left(X_{j}^{k}\right)^{2}}
$$

and the accompanying condition

$$
\sum_{p=1}^{n} X_{p}^{k} \sum_{i=1}^{m} E_{i l} E_{i p}=X_{l}^{k} \sum_{i=1}^{m}\left(T_{i}^{k}\right)^{2} \sum_{p=1}^{n}\left(X_{p}^{k}\right)^{2} .
$$

In order to find solutions for (4) and (5) it is first useful to define the following quantities:

$$
\begin{gathered}
A_{l p}=\frac{1}{m} \sum_{i=1}^{m} E_{i l} E_{i p} \\
\lambda_{k}=\frac{1}{m} \sum_{i=1}^{m}\left(T_{i}^{k}\right)^{2} \sum_{p=1}^{n}\left(X_{p}^{k}\right)^{2}
\end{gathered}
$$

and

$$
\mathbf{X}^{k}=\left(X_{1}^{k}, X_{2}^{k}, X_{3}^{k}, \ldots \ldots, X_{n}^{k}\right)^{T}
$$

where $\frac{1}{m}$ is an introduced normalization factor; $A_{l p}$ is a covariant relationship between the grid points $l$ and $p$; and $\lambda_{k}$ is an eigenvalue. Equation (5) can now be expressed in matrix form as,

$$
\mathbf{A} \mathbf{X}^{k}=\lambda_{k} \mathbf{X}^{k}
$$

and is soluble as a standard eigenvalue problem. The natural orthogonal basis then comprises the full set of eigenmodes $\mathbf{X}^{k}$ and (4) can be used to calculate the temporal coefficients $T_{i}^{k}$.

\subsection{Data Set: UVI Images}

[24] The UVI data set comprises 10,576 images at a spatial resolution of 1 hour MLT by $1^{\circ}$ MLAT down to a lowest latitude of $60^{\circ}$. Using the terminology introduced above, the number of spatial grid points is $n=31 \times 24=$
744 and the number of time steps is $m=10,576$. The variable spatial coverage introduces a complication when applying the MNOC procedure to UVI images. This is not a problem in the calculation of the correlation matrix, $\mathbf{A}$, because missing data can simply be ignored at this step. However, the calculation of the temporal coefficients, $T_{i}^{k}$, requires missing auroral brightness to be estimated in some manner. This has been accomplished by using average images organized by $\mathrm{AE}$ index, IMF, and solar wind. A cumulative point-to-point deviation across the UVI field of view is used to select the appropriate average image that best matches the current auroral conditions. The average image is then used to estimate the auroral brightness outside the UVI field of view and produce a complete image.

\subsection{Results}

[25] The MNOC procedure outlined in section 5.1 is now applied to the data set of UVI images during January 1997. The resulting eigenmodes and eigenvalues are discussed in section 5.3.1 and the temporal coefficients are discussed in sections 5.3.2 and 5.3.3.

\subsubsection{Eigenvalues and Eigenmodes}

[26] Figure 5 plots the UVI eigenvalues, $\lambda_{k}$, for orders $k=0-20$. The leading eigenvalue, $\lambda_{0}=10,729.3$, clearly dominates over higher orders. The next 3 eigenvalues are 1325.8, 845.6, and 507.9. Figure 6 displays the first nine eigenmodes in Apex MLT-MLAT coordinates contoured according to the normalized color scale at the right. Regions in which the eigenmodes are colored red (blue) represent a brightening (quenching) of auroral activity in that particular location. Some of the lower-order eigenmodes can be interpreted in terms of well understood auroral phenomenologies. Most notably, $k=0$ can be associated with the brightness of the main auroral oval, as might be expected from the dominant eigenmode. Likewise, some of the higher orders can be interpreted in terms of secondary auroral processes. For example, $k=1$ shows characteristics of a poleward contraction of the auroral oval, perhaps during northward IMF or substorm expansion and recovery, while $k=2$ is suggestive of the dawn-dusk asymmetry in the auroral oval produced by preferential pitch angle scattering of eastward drifting electrons in the dawn sector. Some of the other eigenmodes can be interpreted in similar ways and this will become clearer when the temporal coefficients for the eigenmodes are correlated against interplanetary parameters in section 5.3.3.

[27] Such associations between eigenmodes and auroral phenomenologies are obviously useful because they allow for the possibility of separating out particular auroral modes for detailed study. However, care should be taken when interpreting the eigenmodes in this way because some auroral behaviors may be represented by combinations of eigenmodes that by themselves are not phenomenologically significant. Indeed, with increasing order the eigenmodes become increasingly localized in character and less easy to interpret physically. With these caveats in mind, it is therefore worthwhile to check the robustness of the eigenmodes to changes in the data. This was done by repeating the eigenmode calculation using only even days during January 1997. The result (not shown) was that the first four eigenmodes were remarkably robust to changes in the data but higher orders were not. For $k=4$ and $k=5$ the 


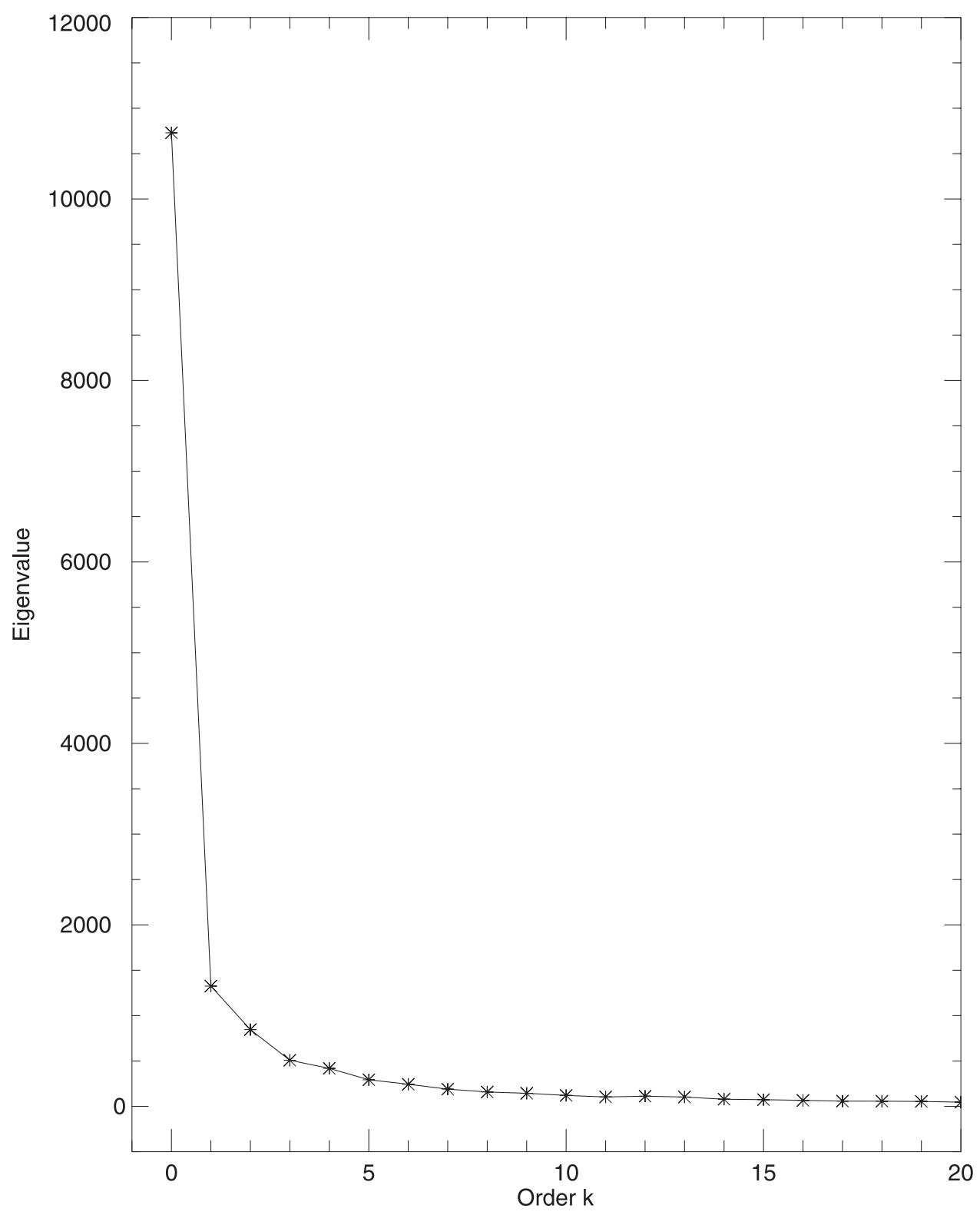

Figure 5. First 20 MNOC eigenvalues for UVI images during January 1997.

eigenmodes retained the majority of their character, particularly on the dayside, but showed minor morphological changes on the nightside and a mixing of features between these two orders. The higher orders $(k>5)$ showed major changes suggesting that they probably represent less significant geophysical influences or statistical noise.

[28] Another useful test is to separate the data according to magnetospheric activity. Figure 7 shows the results when the eigenmode analysis was applied using only UVI images taken when the proxy AE index was less than $200 \mathrm{nT}$. The $k=0$ eigenmode in Figure 7 shows an auroral oval that is thinner, contracted poleward, and relatively brighter on the dayside and at the flanks, particularly in the dawn sector. More generally, there is some mixing of features between orders when one compares Figures 6 and 7. For example, $k=4$ in Figure 7 has characteristics that are a mixture of $k=3$ and $k=4$ in Figure 6. However, the most significant difference between the two figures is a new day-night asymmetry eigenmode $(k=1)$ in Figure 7 that does not exist in Figure 6. This is the dominant secondary auroral process during weak geomagnetic activity and is probably associated with dayside auroral brightenings associated with viscous coupling during northward IMF, as well as nightside pseudo-onset activity during the substorm growth phase. In the analysis that follows, it is the eigenmodes of Figure 6 that are used, rather than those of Figure 7.

\subsubsection{Temporal Coefficients}

[29] Having determined the complete set of auroral eigenmodes in the previous section, the UVI image sequence for the entire month of January 1997 can now be expressed in terms of the temporal coefficients, $T_{i}^{k}$, using (4). In particular, one can monitor the time series of temporal coefficients during active periods in an effort to better understand the physical meaning of the auroral eigenmodes. An example is shown in Figure 8 during the passage of an interplanetary shock past the magnetosphere early on 10 

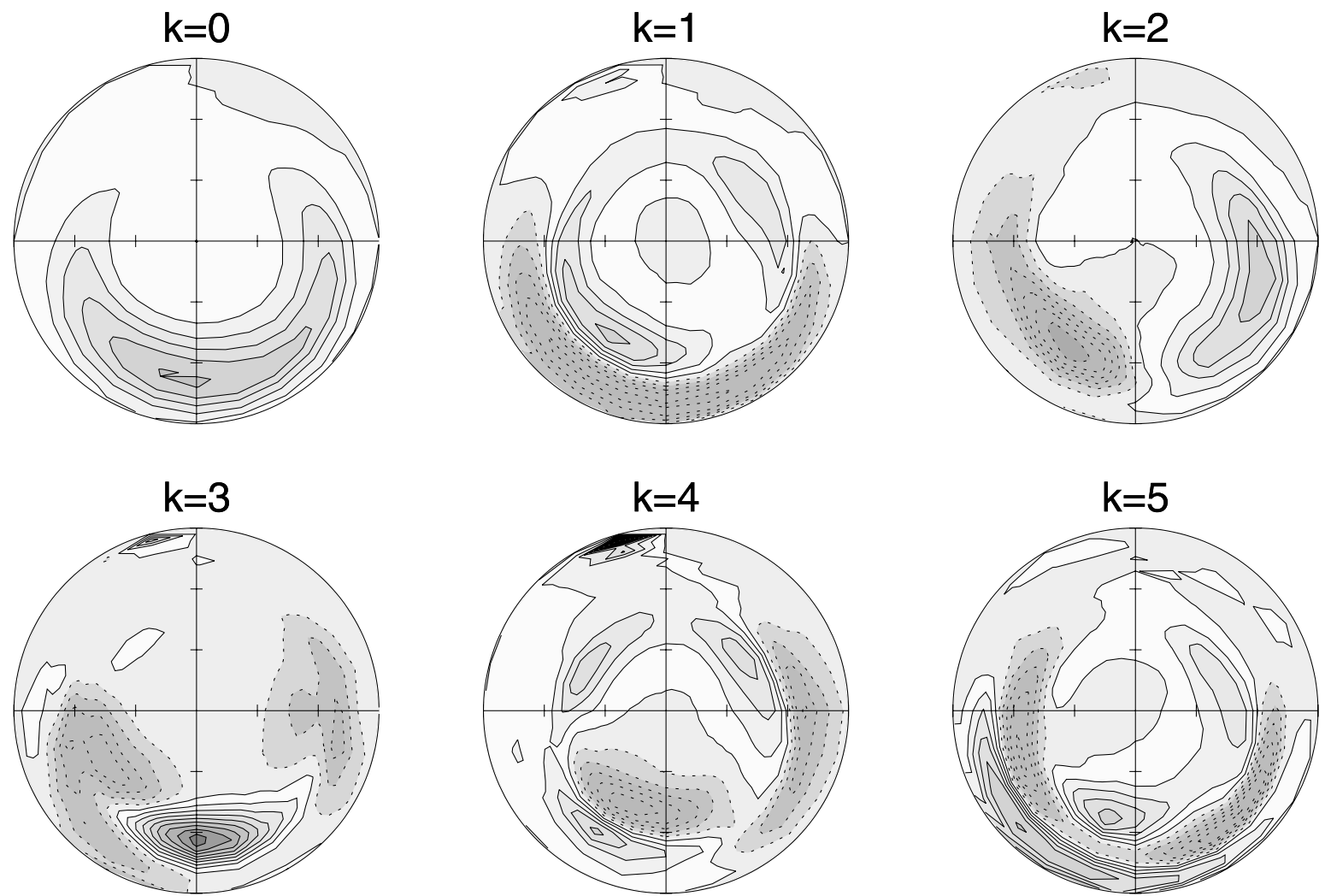

0.1
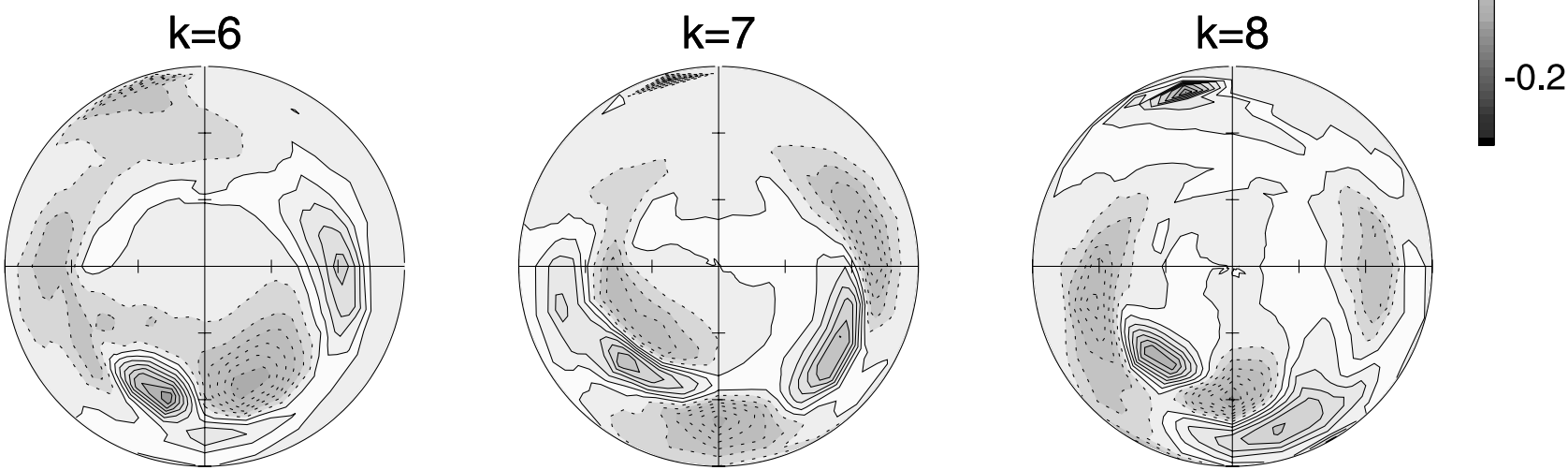

Figure 6. First 9 orthonormal MNOC eigenmodes for UVI images rendered in Apex MLAT-MLT coordinates. Regions of auroral enhancement (quenching) are colored red (blue) according to the normalized color scale at right. See color version of this figure at back of this issue.

January 1997 (see the study of Spann et al. [1998] for more detail of this event). The parameters are presented in a stack plot format with a common horizontal universal time (UT) axis. The upper plot (a) shows the maximum auroral brightness measured by UVI along a magnetic meridian as a function of UT (horizontal axis) and Apex MLT (vertical axis). Auroral photon flux in units of photons $/ \mathrm{cm}^{2} / \mathrm{s}$ is represented according to the gray scale at the right. White pixels are low intensity data rather than missing data. A vertical cut through this plot at a particular UT time yields information from a single UVI image. This format provides a concise means of displaying an extended UVI image sequence. The lower plots are (from top to bottom): MNOC temporal coefficients for the first six orders (b) $-(\mathrm{g})$, solar wind density (h), solar wind bulk speed (i), and IMF $B_{z}$ component $(j)$. The interplanetary parameters have been shifted by a transit time of 14 min from the GSM location of the Wind satellite $(85,-55,-23) R_{E}$ to the ionosphere. This transit time was determined by keying the impulsive increase in auroral activity (0005 UT) to the discontinuity in the solar wind density (2351 UT).

[30] The bottom three plots (h) $-(\mathrm{j})$ in Figure 8 show the solar wind $N, V_{x}$, and IMF $B_{z}$ during the event interval. Starting at just after 0100 UT there was a sudden increase in all 3 parameters signifying the arrival of the interplanetary shock at the magnetopause. The uppermost plot (a) shows that the initial auroral response to the shock was a brightening of the auroral oval on the dayside that propagated to the nightside through dawn and dusk (note the characteristic butterfly wing shape). The response of the first 6 eigenmode 

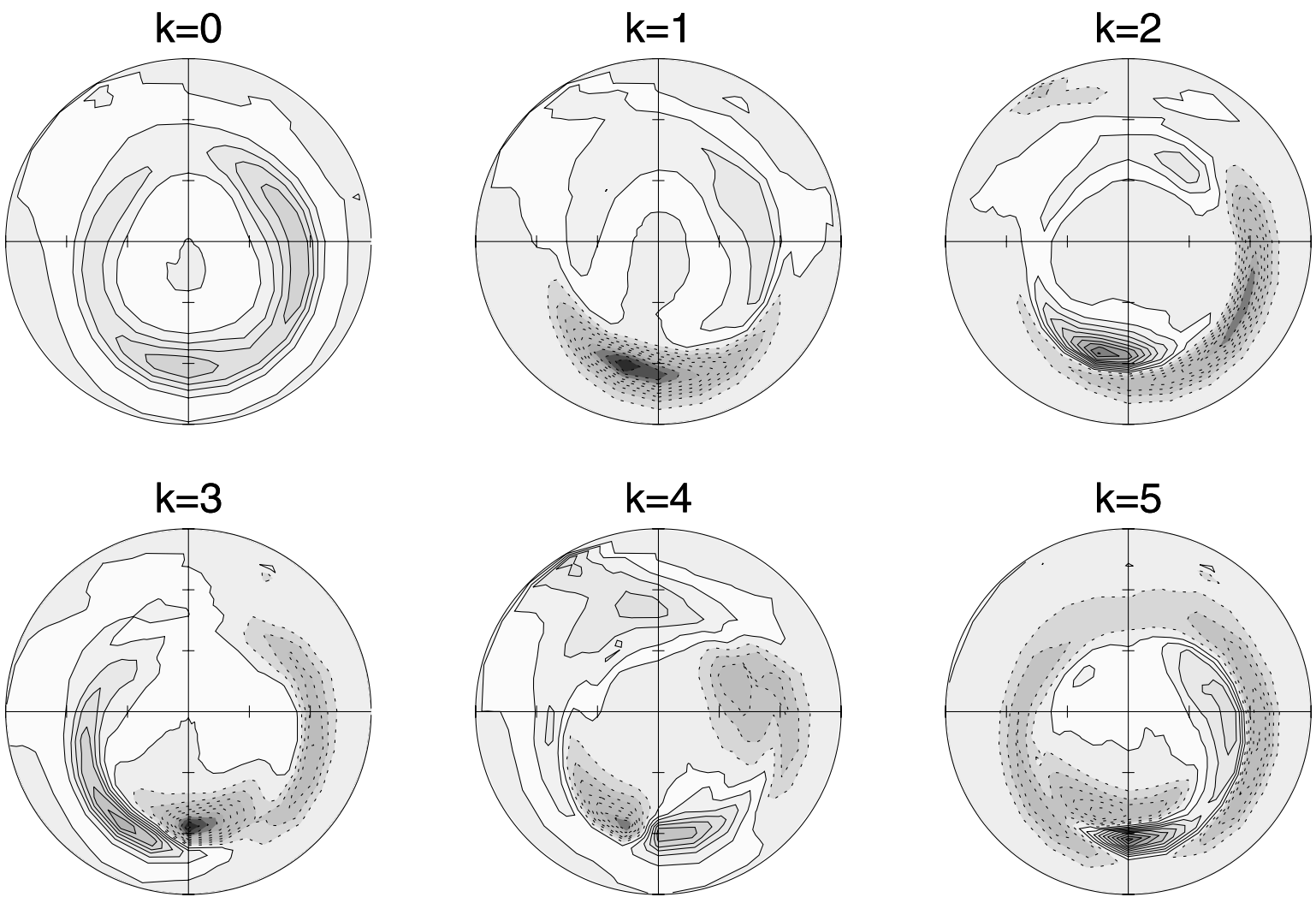

0.1
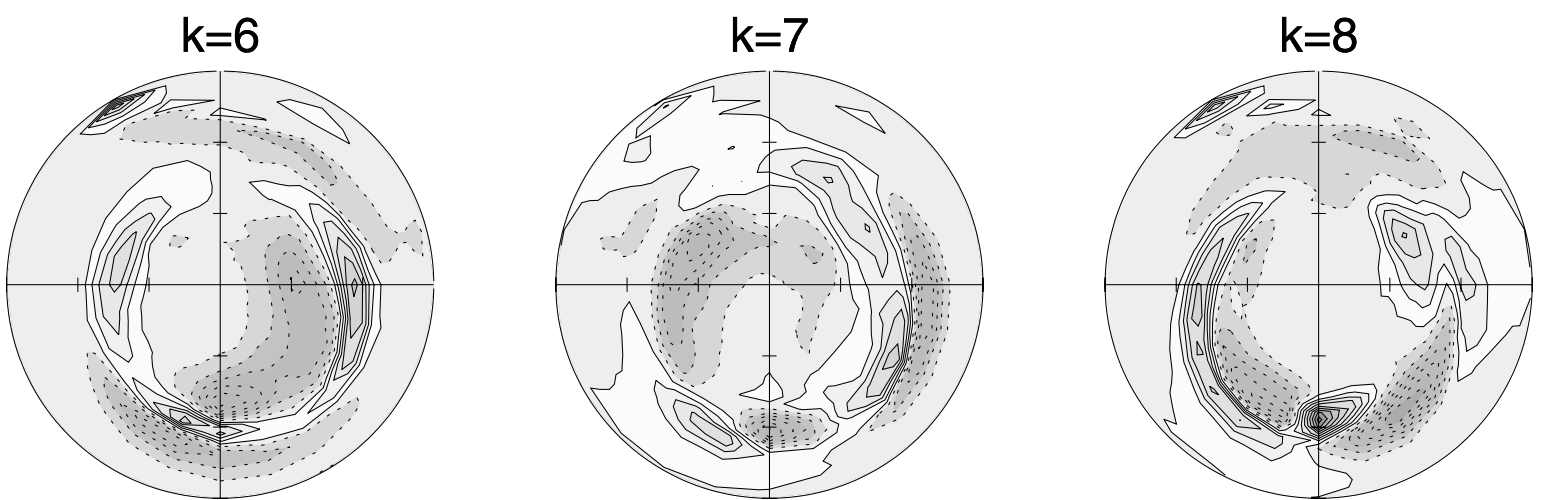

Figure 7. First 9 orthonormal MNOC eigenmodes for UVI images taken during periods for which $\mathrm{AE}<200 \mathrm{nT}$ (in same format as Figure 6). See color version of this figure at back of this issue.

temporal coefficients during this interaction can be examined in detail in $(\mathrm{b})-(\mathrm{g})$. For example, it can be seen that all but one of the temporal coefficients initially increased during the magnetospheric compression, as might be expected. Only the $T^{3}$ coefficient decreased. Figure 6 shows that the $k=3$ eigenmode has the form of an auroral enhancement at midnight. A relative decrease in $T^{3}$ is thus consistent with the midnight auroral gap shown in Figure 8a that existed immediately following the shock impact.

[31] After the initial shock encounter the IMF remained northward for approximately $70 \mathrm{~min}$. Auroral activity during this period was characterized by intense polar cap arcs [see Spann et al., 1998] as well as sporadic midnight sector auroral activations that can be seen in Figure 8a. At approximately $0215 \mathrm{UT}$, the IMF turned southward producing a quick decline in polar cap arc activity and the initiation of a substorm growth phase. Figure $8 \mathrm{a}$ shows that the main auroral onset was at approximately 0340 UT and was preceded by an interval of increased dawnside auroral activity. In this analysis we are less interested in identifying the trigger for the expansion phase, than we are in understanding the behavior of the eigenmodes immediately before and after the onset. Examination of the $T^{1}$ and $T^{5}$ time series shows that both coefficients decreased gradually during the hour preceding onset. Negative trends in the influence of both these eigenmodes is consistent with auroral behavior expected during the substorm growth phase. Namely, an expansion of the polar cap area $(k=1)$ and a simultaneous thinning of the auroral oval $(k=5)$, respectively.

[32] A particularly interesting distinction between the temporal coefficients is their behavior during the onset of the expansion phase at 0340 UT. $T^{2}$ and $T^{5}$ are the only 

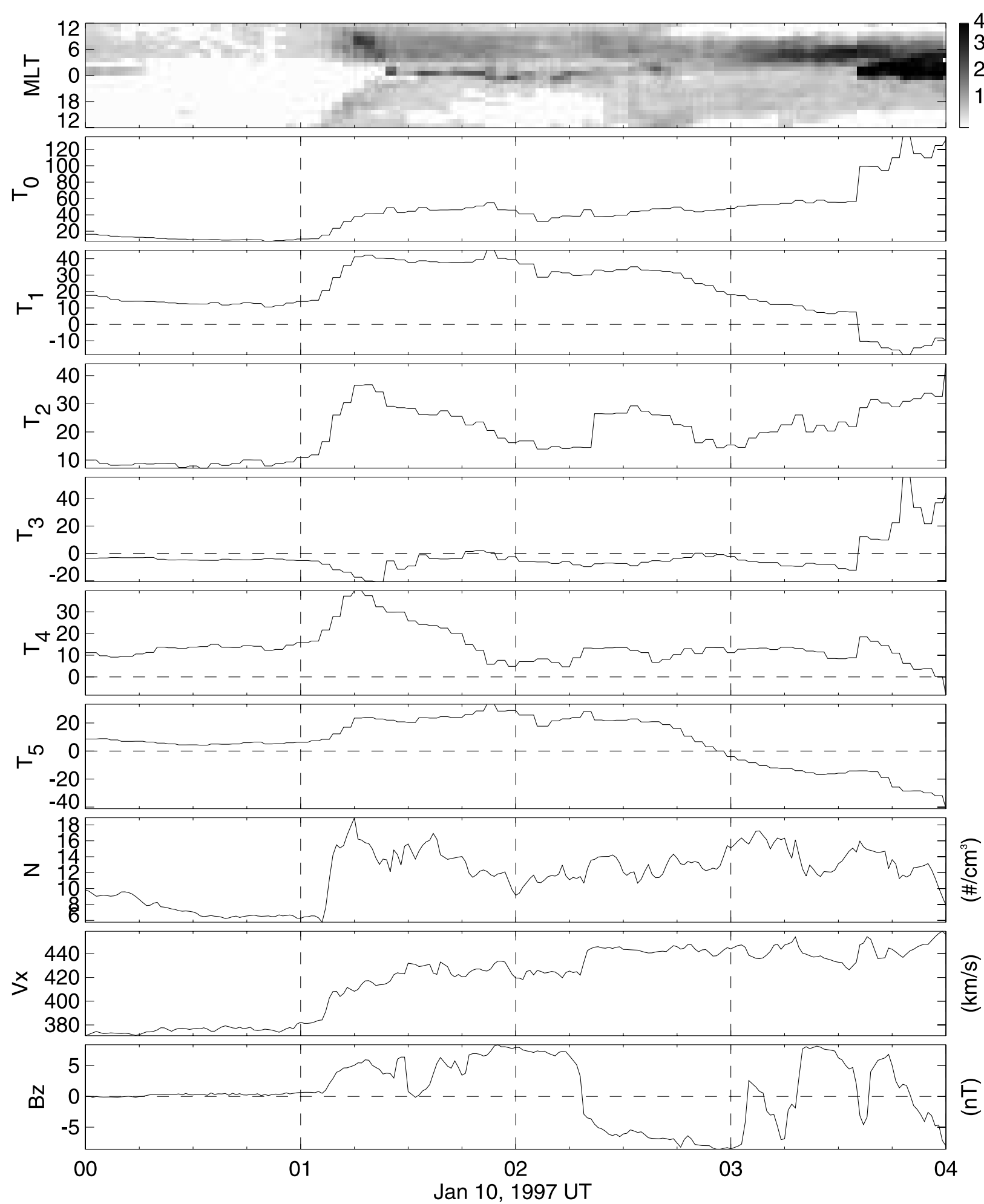

Figure 8. UVI and interplanetary parameters during interplanetary shock on 10 January 1997. Horizontal axis is UT. Panels from top to bottom: (a) maximum UVI meridional auroral brightness versus MLT, (b) - (g) MNOC temporal coefficients $T^{0}-T^{5}$, (h) solar wind $N$, (i) $V_{x}$, and (j) IMF $B_{z}$.

coefficients that do not have substantial discontinuous changes at the time of onset. This suggests that these two eigenmodes are dominated by directly driven auroral activity while the other components include significant unload- ing characteristics. The unloading character of $T^{3}$ is particularly pronounced and is perhaps to be expected because the $k=3$ eigenmode has the form of a midnight sector auroral brightening. One of the advantages of the 

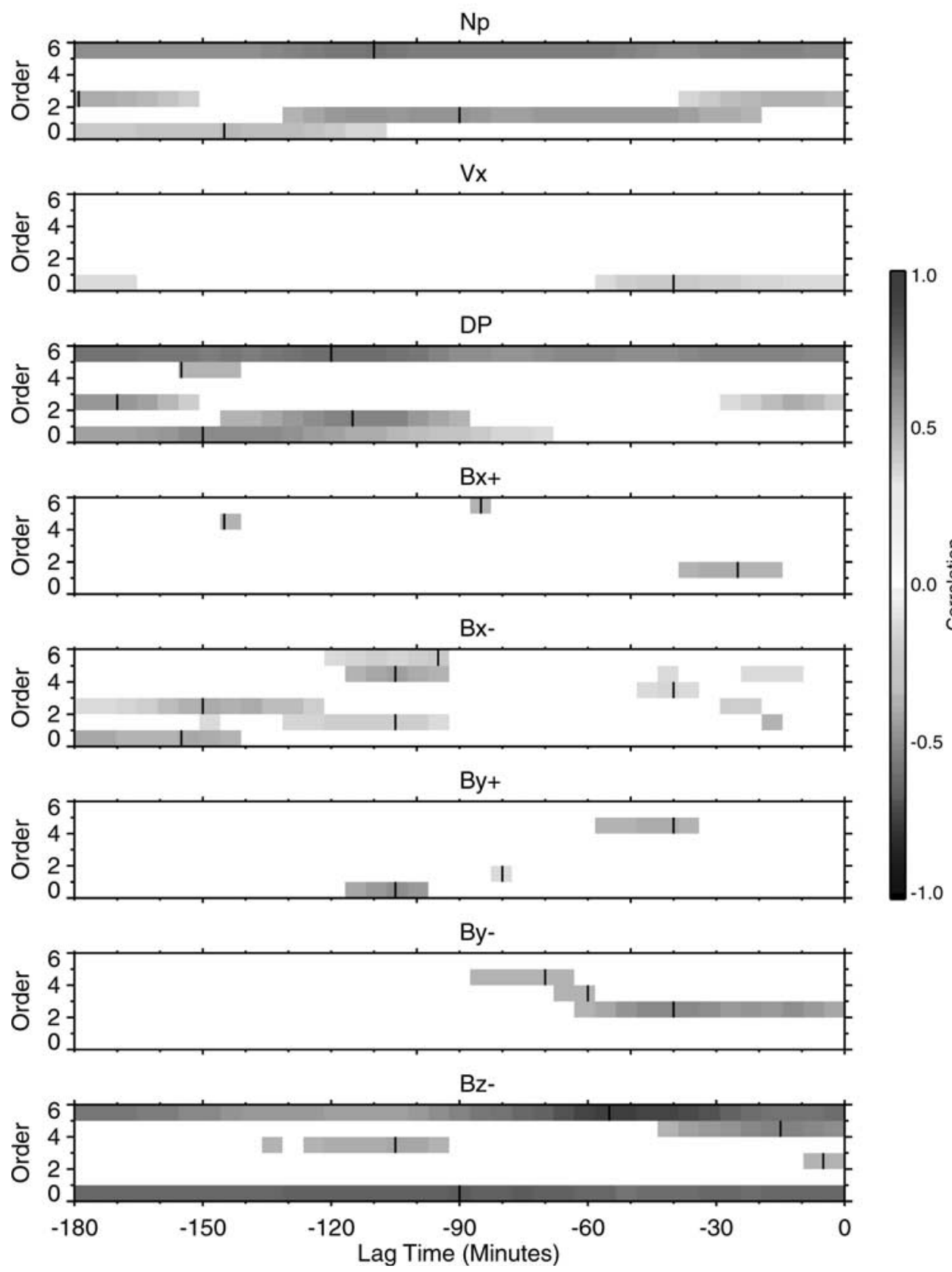

Figure 9. Correlation of MNOC temporal coefficients (vertical axis) against interplanetary parameters during southward IMF with a variable time lag (horizontal axis). Positive (negative) correlations are colored red (blue) according to the scale at right. See color version of this figure at back of this issue.

MNOC technique is that it allows directly driven and loading-unloading components of auroral activity to be investigated separately.

\subsubsection{Correlation With Interplanetary Parameters}

[33] In this section, the first 6 eigenmode temporal coefficients are correlated with interplanetary parameters for southward and northward IMF throughout January 1997. To minimize errors from estimating missing data from binned images only UVI images that have greater than $87 \%$ coverage of the area bounded by the $60^{\circ}$ MLAT are included in this analysis. This subset amounts to a total of 3251 images. UVI images with this amount of spatial coverage tend to be missing information only on the dayside at latitudes below the auroral zone (see Figure 1). Estimation of the aurora in this region with average images is not expected to greatly affect the calculation of the temporal coefficients because they are macroscopic quantities. In addition, the restrictions on the interplanetary parameters displayed in Table 2 are also applied here. All correlation results were tested for robustness by varying the values of the parameters in Table 2 and by arbitrarily removing large sections of the data. This was found to produce minimal changes to the form of the results.

[34] Figures 9 and 10 display the correlation of the MNOC temporal coefficients $T_{i}^{k}$ with interplanetary parameters during southward and northward IMF respectively. 

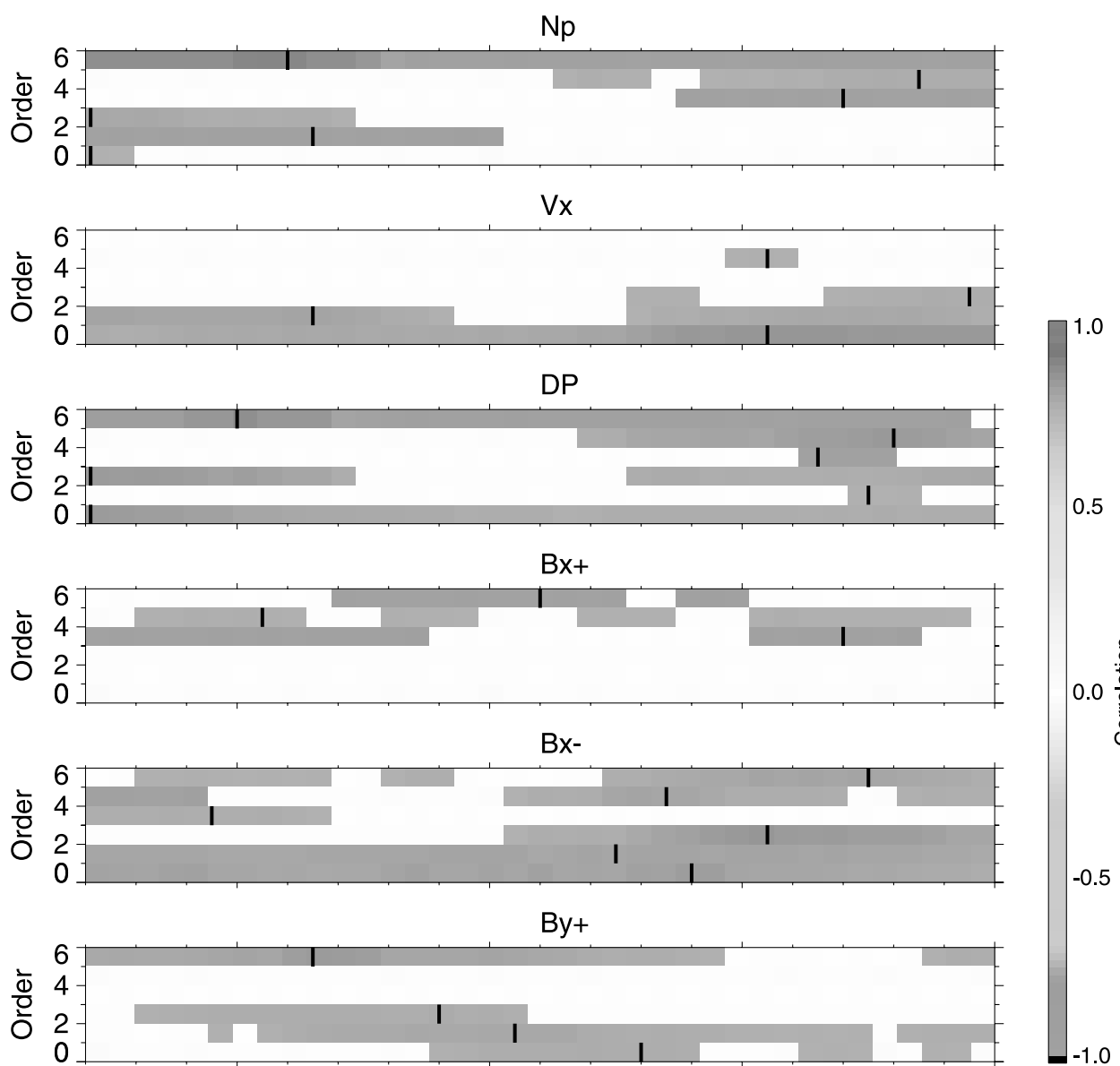

By-

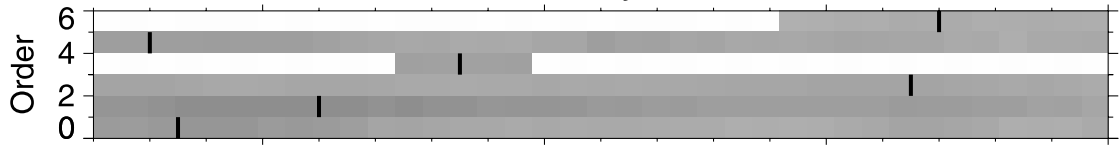

$\mathrm{Bz}+$

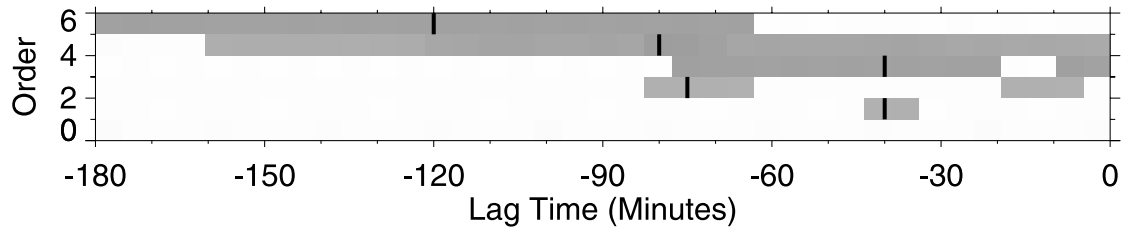

Figure 10. Correlation of $M N O C$ temporal coefficients against interplanetary parameters during northward IMF (in same format as Figure 9). See color version of this figure at back of this issue.

The parameters from top to bottom are solar wind $N, V_{x}$, and dynamic pressure, positive and negative values of IMF $B_{x}$ and $B_{y}$, and IMF $B_{z}$. The vertical axis measures MNOC order, while the horizontal axis measures lag between the time series of the relevant interplanetary parameter and the aurora in minutes. The linear correlation coefficient is represented according to the color scale at the right. Positive correlations are yellow-red, negative correlations are greenblue. Any correlations with magnitudes less than 0.35 have been set to zero to highlight the stronger correlations. Small vertical bars identify the lag times at which the magnitude of the correlations are a maximum for each order.

\subsubsection{Southward IMF}

[35] The dominant correlation in Figure 9 is that between negative IMF $B_{z}$ and $T^{0}$ with an optimal lag time of $90 \mathrm{~min}$ (bottom panel). However, the time dependence of this correlation is very weak, suggesting that the negative $B_{z}$ influence operates over a wide variety of timescales. Negative $B_{z}$ also has short timescale anticorrelations with $T^{2}$ and $T^{4}$. Figure 6 shows that these eigenmodes have auroral features at dawn $(k=2)$ and on the dayside $(k=4)$ respectively, where viscous interactions might be expected to be important. Short timescale anticorrelations with these two eigenmodes can thus be interpreted as a relative decrease in the influence of viscous interactions when the IMF turns southward. Also noteworthy is an anticorrelation with $T^{5}$ on a timescale of $50 \mathrm{~min}$. This is consistent with a thinning of the auroral oval during the substorm growth phase, as was seen in Figure 8. By contrast, the dependence of the auroral eigenmodes on other interplanetary parame- 
Table 3. Correlation of MNOC Eigenmodes With Interplanetary Parameters for Southward IMF

\begin{tabular}{lcccc}
\hline Order & Parameter & $\mathrm{C}$ & $\mathrm{T}(\mathrm{min})$ & Auroral Processes \\
\hline$k=0$ & $B_{y}+$ & -0.49 & 105 & Main auroral oval \\
& $B_{z}-$ & 0.78 & 90 & \\
& $N$ & 0.46 & 145 & \\
$k=1$ & $N$ & -0.48 & 90 & Auroral bulge \\
$k=2$ & $B_{x}-$ & 0.51 & 150 & Dawn/dusk aurora \\
& $B_{y}-$ & -0.51 & 40 & \\
$k=3$ & $N$ & 0.51 & 180 & \\
& - & - & - & Auroral bulge \\
$k=4$ & - & - & - & Midnight gap \\
$k=5$ & $B_{z}-$ & -0.53 & 15 & Nightside aurora \\
& $B_{z}-$ & -0.75 & 55 & Oval thinning \\
& $N$ & -0.60 & 110 & Double oval \\
\hline
\end{tabular}

ters during southward IMF is much weaker. The asymmetry between positive and negative values of $B_{x}$ and $B_{y}$ identified in Figures 3 and 4 is not readily apparent in Figure 9. Negative $B_{y}$ has an anticorrelation with $T^{2}$ (dawn-dusk asymmetry) at a time lag of $0-45 \mathrm{~min}$, suggesting that it is associated with a skewing of the auroral oval toward the premidnight sector. The combined correlations with negative $B_{x}$ suggest that it has some influence over the aurora on short and long timescales that may be consistent with preferential antiparallel merging in the Northern Hemisphere.

[36] The upper three panels in Figure 9 show the influence of the solar wind $N, V_{x}$, and $P$ during southward IMF. The strongest correlations are with $P$ at very long timescales. The similarities between the $P$ and $N$ panels show that the dependence on $P$ is dominated by the influence of $N$ rather than $V_{x}$. These long timescale correlations are undoubtedly connected with substorm phenomena and suggest that the solar wind density plays a prominent role in preparing the magnetosphere for substorm expansion, possibly by providing a source population for the inner plasma sheet and/or the necessary lobe pressure to initiate reconnection in the plasma sheet. In addition, both $N$ and $V_{x}$ show significant correlations on shorter timescales. $V_{x}$ is correlated with $T^{0}$ over $0-60 \mathrm{~min}$, suggesting that auroral activity in the main auroral oval is in part directly driven by the solar wind electric field or the Kelvin-Helmholtz instability. Short timescale correlations between $N$ and higher-order eigenmodes $\left(T^{1}, T^{2}\right.$, and $\left.T^{5}\right)$ are consistent with an expanded polar cap, a thinned auroral oval, and an increase in dawnside aurora during periods of high solar wind density.

\subsubsection{Northward IMF}

[37] The correlation results in Figure 10 show a much richer dependence of auroral behavior on interplanetary parameters during northward IMF. The dominant influence of the $B_{z}$ component over the $k=0$ eigenmode vanishes and positive $B_{z}$ is instead correlated with a collection of higherorder eigenmodes (particularly $k=4$ ) that interpreted together are consistent with a weak contracted auroral oval, much like $k=0$ in Figure 7. The $B_{x}$ and $B_{y}$ components (particularly negative values) have a much greater influence over the shape of the auroral distribution during northward IMF. The weak time dependence of these correlations are reminiscent of the $B_{z}$ dependence over $k=0$ in Figure 9. These results suggests that magnetic reconnection does not cease when the IMF turns northward, but is reduced significantly and becomes more dependent on the detailed $B_{x} / B_{y}$ geometry.

[38] One is also struck by the increased influence of the solar wind plasma parameters when the IMF turns northward (upper three panels), particularly at short timescales. This is consistent with a relative increase in the significance of viscous interactions during northward IMF. However, there still remain significant correlations at long timescales, suggesting that $N$ and $V_{x}$ maintain some control over auroral activity on substorm timescales. Of particular interest is a dramatic increase in the influence of the solar wind velocity at long timescales. This can be interpreted in terms of high latitude reconnection. When the reconnection site moves away from the subsolar stagnation region the reconnection rate becomes more dependent on $V_{x}$ because it controls the rate at which magnetic flux can be transported to the reconnection site.

\section{Discussion}

[39] An understanding of the influences that the solar wind and IMF have over the aurora is complicated by a mixture of directly driven and magnetotail unloading processes that operate over variable temporal and spatial scales. Dayside auroral transients are driven by interplanetary influences on timescales of a few minutes [e.g., Sitar et al., 1998], whereas the integrated nightside auroral power responds to a southward turning of the IMF on a timescale of $\sim 60 \mathrm{~min}$ [Liou et al., 1998]. This paper has examined how the aurora responds to the solar wind and IMF throughout the entire polar region as a function of space and time. Two techniques have been used: a traditional time-lagged linear correlation analysis (section 4) and the MNOC procedure (section 5). Table 3 summarizes the most prominent MNOC correlation results for southward IMF and provides phenomenological interpretations for the first six eigenmodes. Table 4 summarizes the MNOC correlation results for northward IMF.

[40] Gussenhoven [1988] described magnetospheric activity in terms of three basic states parameterized by

Table 4. Correlation of MNOC Eigenmodes With Interplanetary Parameters for Northward IMF

\begin{tabular}{cccc}
\hline Order & Parameter & $\mathrm{C}$ & $\mathrm{T}(\mathrm{min})$ \\
\hline$k=0$ & $B_{x}-$ & 0.67 & 60 \\
& $B_{y}-$ & 0.73 & 165 \\
& $V_{x}$ & 0.74 & 45 \\
& $B_{x}-$ & 0.63 & 75 \\
& $B_{y}-$ & 0.80 & 140 \\
& $B_{y}+$ & 0.57 & 95 \\
& $N$ & -0.54 & 135 \\
& $V_{x}$ & 0.62 & 135 \\
$k=1$ & $B_{x}-$ & 0.72 & 45 \\
& $B_{y}-$ & 0.62 & 35 \\
& $N=35$ & 180 \\
$k=3$ & $B_{x}-$ & 0.50 & 155 \\
& $B_{x}-$ & 0.63 & 65 \\
& $B_{y}-$ & 0.68 & 170 \\
$k=5$ & $B_{z}+$ & 0.67 & 80 \\
& $B_{x}-$ & 0.62 & 25 \\
& $B_{y}-$ & 0.51 & 30 \\
& $B_{y}+$ & 0.70 & 135 \\
& $N$ & -0.75 & 140 \\
\hline
\end{tabular}


IMF $B_{z}$ : the active auroral state ( $B_{z}$ negative), the active polar cap state ( $B_{z}$ positive), and the quiet state $\left(B_{z}\right.$ zero). The results presented here confirm the dominant influence of the IMF $B_{z}$ component over a wide range of timescales. Negative $B_{z}$ has a strong linear correlation with the main auroral oval brightness that maximizes at an optimal time lag of $90 \mathrm{~min}$. However, this correlation has a very weak time dependence, probably due to the loadingunloading nature of substorm phenomena. This result suggests that there is a highly variable timescale for the substorm dynamic cycle extending well beyond 2-3 hours in some cases. When the IMF remains southward for an extended period of time the auroral distribution becomes dominated by the primary auroral eigenmode and resembles a steady magnetospheric convection event. However, this rarely happens. Changes in the $B_{z}$ component and the influence of other interplanetary parameters introduce higher-order modes of auroral activity that are generally ubiquitous and add character and subtlety to the auroral distribution.

[41] As noted in the introduction, a number of recent studies have utilized UVI auroral images to identify an IMF $B_{y}$ asymmetry in the Northern Hemisphere aurora [Liou et al., 1998; Shue et al., 2001]. The correlation maps presented here confirm that the aurora does indeed have a higher correlation with negative $B_{y}$ than it does with positive $B_{y}$. The MNOC results confirmed this during northward IMF but not southward IMF. It has also been suggested that the $B_{x}$ component might be responsible for interhemispheric asymmetries in magnetopause reconnection and an associated Northern Hemisphere auroral bias during negative $B_{x}$ [Cowley, 1981b; Crooker et al., 1985]. The results of this paper confirm that the northern auroral distribution does indeed have a higher correlation with negative $B_{x}$ than it does with positive $B_{x}$, consistent with the recent findings of Shue et al. [2002].

[42] Liou et al. [1998] found that the solar wind density did not show a consistent linear correlation with the integrated nightside auroral power in UVI images. The correlation maps for $N$ in Figures 3 and 4 are consistent with this picture. However, the MNOC results in Figures 9 and 10 showed that $N$ is actually well correlated with many eigenmodes of auroral activity. Furthermore, the wide variety of timescales for these correlations suggest that $N$ plays a role in the generation of both directly driven and loading-unloading auroral activity. A directly driven influence can be understood in terms of pressure-induced viscous interactions at the magnetopause that influence the higher-order eigenmodes, particularly during northward IMF. An influence over loading-unloading activity can perhaps be explained in terms of $N$ acting as a source population for the inner plasma sheet on a timescale of $2-3$ hours. These findings highlight an important advantage of using the MNOC procedure. Namely, it allows subtle relationships between the aurora and interplanetary parameters to be isolated from the dominant influence of the IMF $B_{z}$ component.

[43] Some experimentation was done to test how robust the correlation results in sections 4.1 and 5.3.3 were to restricting the UVI databased on the $\mathrm{AE}$ index. In both cases, the results (not shown) were essentially unchanged during northward IMF, but there were some changes to the magnitudes of the correlation coefficients and the optimal lag times during southward IMF. The differences were more dramatic for the correlation maps in section 4.1 than they were for the MNOC results in section 5.3.3. This is because the methodology used in section 4.1 is far too simplistic. The MNOC analysis is able to distinguish a number of overlapping modes of auroral activity that can operate on multiple timescales at a single location in space. The correlation maps tend to mix these influences together. Another advantage of the MNOC analysis is that it condenses the global UVI auroral information into a handful of macroscopic measures of auroral activity that can be manipulated and displayed economically, as was done in Figure 8.

[44] However, there is still some ambiguity associated with the fact that the auroral oval contracts and expands in response to geomagnetic conditions. For this reason, it is perhaps not entirely appropriate to perform the MNOC analysis on a static spatial grid. The relative substorm morphology of Gjerloev and Hoffman [2000a, 2000b] provides one possibility for improvement in this regard. In those studies, DE-1 auroral images were organized with respect to key features of the generic bulge-type auroral substorm developed by Fuji et al. [1994]. Six different local time sectors with characteristic substorm emission signatures were used to order the data. A similar approach could be used in conjunction with the MNOC procedure. The auroral information could first be transformed to the relative substorm reference frame before application of the MNOC technique. Such a transformation might be expected to improve the separation of eigenmodes and the fidelity of the correlation results.

[45] The computational cost of the MNOC procedure in itself is slight. The production of eigenmodes was done using the Linear Algebra PACKage (LAPACK) software in Fortran77. The run time on a Linux configured DELL dimension PC with a Pentium III processor was on the order of 30-40 min. The bulk of the computational effort was spent preparing the UVI data for input to the MNOC procedure: processing the raw data and converting it to the MLAT-MLT grid. Further work using the MNOC procedure will involve additional event analysis, such as that done in section 5.3.2, and will extend the MNOC statistical analysis presented here to other seasons. The MNOC analysis would also be ideal for a detailed analysis of the relative merits of the various solar wind-magnetosphere coupling functions that have been postulated over the years.

[46] Finally, some specific comment is required regarding the correlations that maximize at long lag times in Figures 9 and 10. The maximum lag time was set somewhat arbitrarily to $180 \mathrm{~min}$ in order to highlight the behavior of the eigenmodes at low lags. There are substantial correlations that operate on timescales well in excess of this limit, particularly with the higher-order eigenmodes. It is difficult to determine whether these correlations are associated with real physical auroral processes, or rather represent a unique decomposition of the UVI images that should not be interpreted in terms of the individual eigenmodes. However, the fact that most of these correlations are with eigenmodes that include substantial dayside auroral activity is perhaps not coincidental. One possibility is that the long-term 
correlations represent a coupling of the partial ring current to the dayside auroral ionosphere during magnetic storms. If this is the case, then the MNOC analysis might provide a useful framework with which to analyze the relationship between storms and substorms. Detailed event analysis would be required to resolve this question unambiguously, and is beyond the scope of this paper. Such a study will be another focus for future work.

\section{Summary}

[47] The influence of interplanetary parameters on the global auroral distribution measured by UVI images has been investigated using two methods. First, a traditional time-lagged linear correlation analysis as a function of MLAT and MLT has been used to produce spatial maps of maximum magnitude correlation coefficient and optimal lag times. Second, the MNOC has produced a set of orthogonal eigenmodes of auroral activity. The dominant eigenmodes have been interpreted in terms of common auroral phenomenologies: (1) the main auroral oval brightness, (2) polar cap contraction/expansion, (3) dawn/dusk aurora, and (4) midnight sector brightening/quenching. An important advantage of the eigenmode analysis is that it allows some of the more subtle relationships with the solar wind and IMF to be isolated from the dominant influence of the IMF $B_{z}$ component. It also provides an economical framework with which to analyze extended time series of auroral image information.

[48] Acknowledgments. We thank R. P. Lepping, the principal investigator of the MFI experiment, K. W. Ogilvie, the principal investigator of the SWE experiment, and G. K. Parks, the principal investigator of the UVI instrument, for providing data. Support for this work at the University of Michigan has been provided by NASA grant NAG5-9536 and NSF grants OPP-9876473, ATM-9980078, ATM9628706, and ATM-0096283.

\section{References}

Baker, D. N., Statistical analyses in the study of solar wind- magnetosphere coupling, in Solar Wind-Magnetosphere Coupling, edited by Y. Kamide and J. A. Slavin, p. 17, Terra Sci., Tokyo, 1986.

Cowley, S. W. H., Magnetospheric asymmetries associated with the ycomponent of the IMF, Planet. Space Sci., 29, 79, 1981a.

Cowley, S. W. H., Asymmetry effects associated with the X-component of the IMF in a magnetically open magnetosphere, Planet. Space Sci., 29, $809,1981 b$.

Crooker, N. U., J. G. Luhmann, J. R. Spreiter, and S. S. Stahara, Magnetopause merging site asymmetries, J. Geophys. Res., 90, 341, 1985.

Elphinstone, R. D., and D. J. Hearn, The auroral distribution and its relation to magnetospheric processes, Adv. Space Res., 13, 417-427, 1993.

Elphinstone, R. D., K. Jankowska, J. S. Murphree, and L. L. Cogger, The configuration of the auroral distribution for interplanetary magnetic field $B_{z}$ northward, 1 , IMF $B_{x}$ and $B_{y}$ dependencies as observed by the Viking satellite, J. Geophys. Res., 95, 5791, 1990.

Frynberg, E. B., Separation of geomagnetic field into a normal and anomalous part, Geomagn. Aeron., 15, 117, 1975.

Fuji, R., R. A. Hoffmann, P. C. Anderson, J. D. Craven, M. Sugiura, L. A. Frank, and N. Maynard, Electrodynamic parameters in the nighttime sector during auroral substorms, J. Geophys. Res., 99, 6093, 1994.

Germany, G. A., G. K. Parks, M. Brittnacher, J. Cumnock, D. Lummerzheim, J. F. Spann, L. Chen, P. G. Richards, and F. J. Rich, Remote determination of auroral energy characteristics during substorm activity, Geophys. Res. Lett., 24, 995, 1997.

Gjerloev, J. W., and R. A. Hoffman, Height integrated conductivity in auroral substorms, 1, Data, J. Geophys. Res., 105, 215, 2000a.

Gjerloev, J. W., and R. A. Hoffman, Height integrated conductivity in auroral substorms, 2, Modeling, J. Geophys. Res., 105, 227, 2000 b.
Golovkov, V. P., N. E. Papitashvili, Y. S. Tyupkin, and E. P. Kharin, Separation of geomagnetic field variations on the quiet and disturbed components by the mnoc, Geomagn. Aeron., 18, 511, 1978.

Gussenhoven, M. S., Low-altitude convection, precipitation and current patterns in the baseline magnetosphere, Rev. Geophys., 26, 792, 1988.

Jankowska, K., R. D. Elphinstone, J. S. Murphree, L. L. Cogger, and D. Hearn, The configuration of the auroral distribution for interplanetary magnetic field $B_{z}$ northward, 2, Ionospheric convection consistent with Viking observations, J. Geophys. Res., 95, 5805-5816, 1990.

Kendall, M. G., and A. Stuart, The Advanced Theory of Statistics, vol. 3, chap. 35, Charles Griffin, London, 1976.

Lassen, K., and C. Danielson, Quiet time pattern of auroral ares for different directions of the interplanetary magnetic field in the y-z plane, J. Geophys. Res., 83, 5277, 1978.

Lepping, R. P., et al., The WIND magnetic field investigation, Space Sci. Rev., 71, 207, 1995 .

Liou, K., P. T. Newell, C.-I. Meng, M. Brittnacher, and G. Parks, Characteristics of the solar wind controlled auroral emissions, J. Geophys. Res., 103, 17,543, 1998.

Lui, A. T. Y., Characteristics of the cross-tail current in the Earth's magnetotail, in Magnetospheric Currents, Geophys. Monogr. Ser., vol. 28, edited by T. A. Potemra, p. 158, AGU, Washington, D. C., 1984.

Maezawa, K., Statistical study of the dependence of geomagnetic activity on solar wind parameters, in Quantitative Modeling of Magnetospheric Processes, Geophys. Monogr. Ser, vol. 21, edited by W. P. Olsen, p. 436, AGU, Washington, D. C., 1979.

Meng, C.-I., Polar cap variations and the interplanetary magnetic field, in Dynamics of the Magnetosphere, edited by S.-I. Akasofu, p. 23, D. Reidel, Norwell, Mass., 1979.

Murayama, T., Coupling function between solar wind parameters and geomagnetic indices, Rev. Geophys., 20, 623, 1982.

Murphree, J. S., and R. D. Elphinstone, Correlative studies using the Viking imagery, Adv. Space Res., 8, 9, 1987.

Murphree, J. S., C. D. Anger, and L. L. Cogger, The instantaneous relationship between polar cap and oval auroras at times of northward interplanetary magnetic field, Can. J. Phys., 60, 349, 1982.

Murphree, J. S., R. D. Elphinstone, D. Hearn, and L. L. Cogger, Large-scale high-latitude dayside auroral emissions, J. Geophys. Res., 95, 2345, 1989.

Ogilvie, K. W., et al., SWE, a comprehensive plasma instrument for the WIND spacecraft, Space Sci. Rev., 71, 55, 1995.

Papitashvili, N. E., V. O. Papitashvili, B. A. Belov, L. Hakkinen, and C. Sucksdorff, Magnetospheric contribution to k-indices, Geophys. J. Int., 111, 348, 1992.

Papitashvili, V. O., B. A. Belov, D. S. Faermark, Y. I. Feldstein, S. A. Golyshev, L. I. Gromova, and A. E. Levitin, Electric potential patterns in the northern and southern polar regions parameterized by the interplanetary magnetic field, J. Geophys. Res., 99, 13,251, 1994.

Pushkov, A. N., E. B. Frynberg, T. A. Chernova, and M. V. Fiskina, Analysis of the space-time structure of the main geomagnetic field by expansion into natural orthogonal components, Geomagn. Aeron., 16, 196, 1976.

Reiff, R. H., and J. G. Luhmann, Solar wind control of the polar-cap voltage, in Solar Wind-Magnetosphere Coupling, edited by Y. Kamide and J. A. Slavin, p. 453, Terra Sci., Tokyo, 1986.

Richmond, A. D., Ionospheric electrodynamics using magnetic APEX coordinates, J. Geomagn. Geoelectr., 47, 191, 1995.

Rostoker, G., H.-L. Lam, and W. D. Hume, Response time of the magnetosphere to the interplanetary electric field, Can. J. Phys., 50, 544, 1972.

Rotanova, N. M., N. E. Papitashvili, and A. N. Pushkov, Use of natural orthogonal components to distinguish and analyze the 60 -yr geomagnetic field variations, Geomagn. Aeron., 22, 821, 1982.

Shue, J.-H., P. T. Newell, K. Liou, and C.-I. Meng, Influence of interplanetary magnetic field on global auroral patterns, J. Geophys. Res., 106, 5913, 2001.

Shue, J.-H., P. T. Newell, K. Liou, C.-I. Meng, and S. W. H. Cowley, Interplanetary magnetic field $B_{x}$ asymmetry effect on auroral brightness, J. Geophys. Res., 107(A8), 1197, doi:10.1029/2001JA000229, 2002.

Sitar, R. J., J. B. Baker, C. R. Clauer, A. J. Ridley, J. A. Cumnock, V. O. Papitashvili, J. Spann, M. J. Brittnacher, and G. K. Parks, Multi-instrument analysis of the ionospheric signatures of a hot flow anomaly occurring on July 24, 1996, J. Geophys. Res., 103, 23,357, 1998.

Spann, J. F., M. Brittnacher, R. Elsen, G. A. Germany, and G. K. Parks, Initial response and complex polar cap structures of the aurora in response to the January 10, 1997 magnetic cloud, Geophys. Res. Lett., 25, 2577, 1998.

Sun, W., W.-Y. Xu, and S.-I. Akasofu, Mathematical separation of directly driven and unloading components in the ionospheric equivalent currents during substorms, J. Geophys. Res., 103, 11,695, 1998. 
Torr, M. R., D. G. Torr, J. R. Jasperse, and J. A. Whalen, A far ultraviolet imager for the international solar-terrestrial physics mission, Space Sci. Rev., 71, 329, 1995.

Vo, H. B., and J. S. Murphree, A study of auroral bright spots seen by the Viking auroral imager, J. Geophys. Res., 100, 3649-3655, 1995.

Wing, W., P. T. Newell, D. G. Sibeck, and K. B. Baker, A large statistical study of the entry of interplanetary magnetic field y component into the magnetosphere, Geophys. Res. Lett., 22, 2083, 1995.

Zanetti, L. J., T. A. Potemra, R. E. Erlandson, P. F. Bythrow, and B. J. Anderson, Polar region Birkeland current, convection and aurora for northward interplanetary magnetic field, J. Geophys. Res., 95, 58255833, 1990.

J. B. Baker, Applied Physics Laboratory, Johns Hopkins University, 11100 Johns Hopkins Road, Laurel, MD 20723-6099, USA. (bakerjb1@ jhuapl.edu)

C. R. Clauer, V. O. Papitashvili, and A. J. Ridley, Space Physics Research Laboratory, University of Michigan, Ann Arbor, MI, USA 


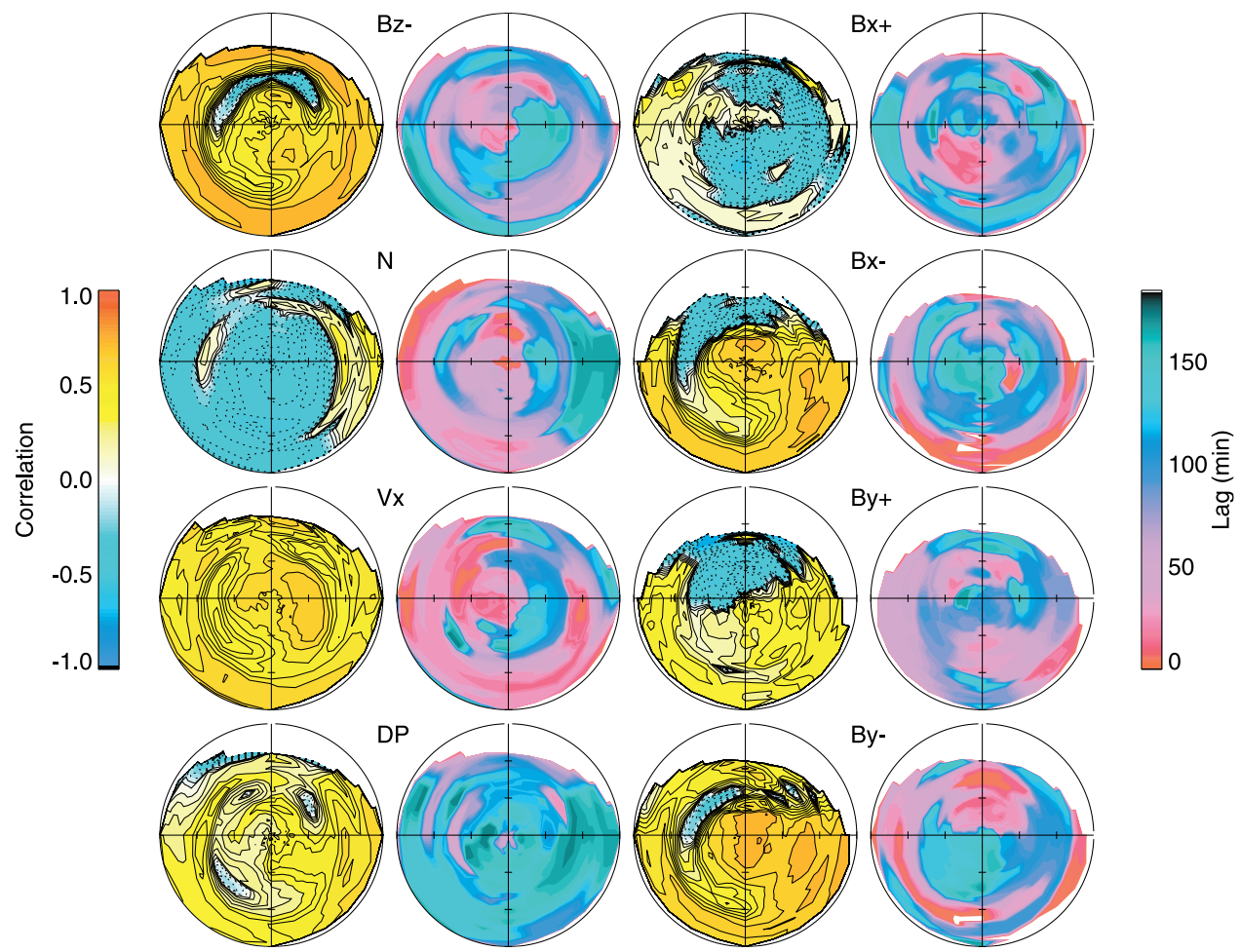

Figure 3. Contours of maximum magnitude correlation (columns 1 and 3; left color scale) and optimal lag time (columns 2 and 4; right color scale) between interplanetary parameters and UVI-measured aurora during southward IMF. Representation is in Apex MLAT-MLT coordinates down to a lowest latitude of $60^{\circ}$ with magnetic noon at the top.

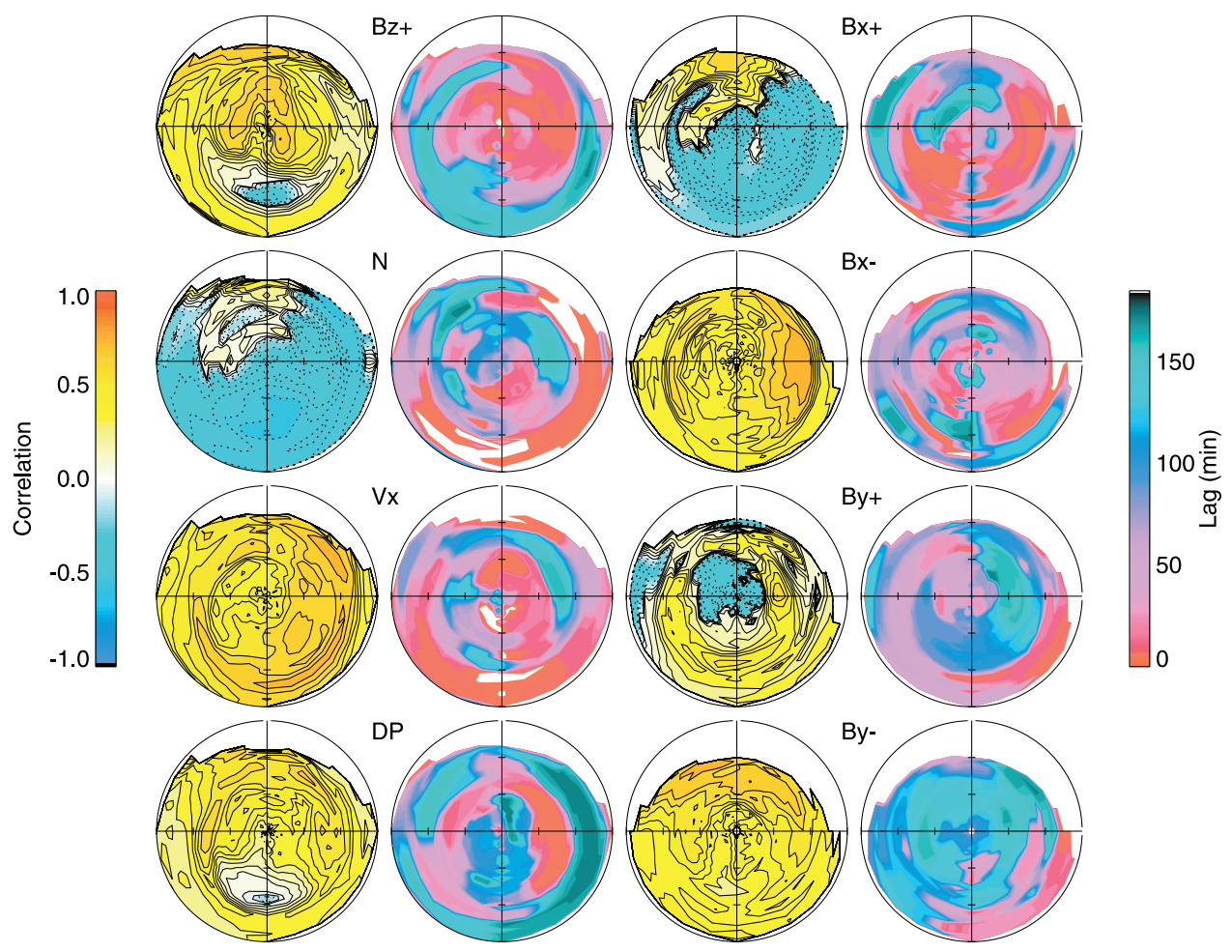

Figure 4. Contours of maximum magnitude correlation and optimal lag time between interplanetary parameters and UVI-measured aurora during northward IMF (in same format as Figure 3). 

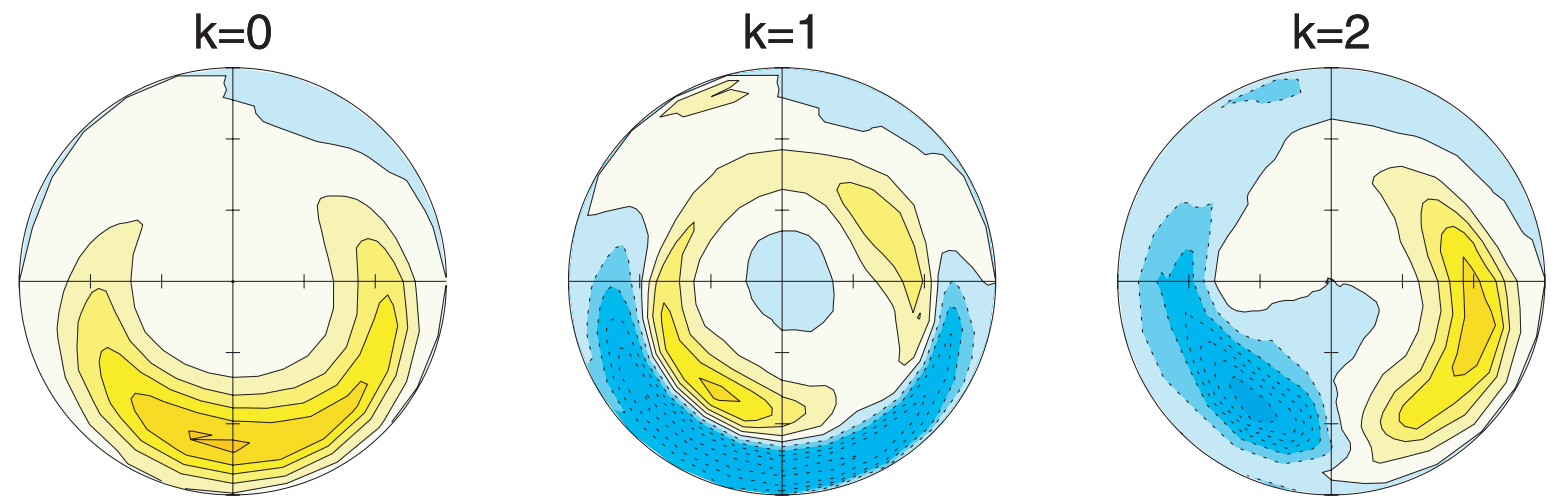

0.2
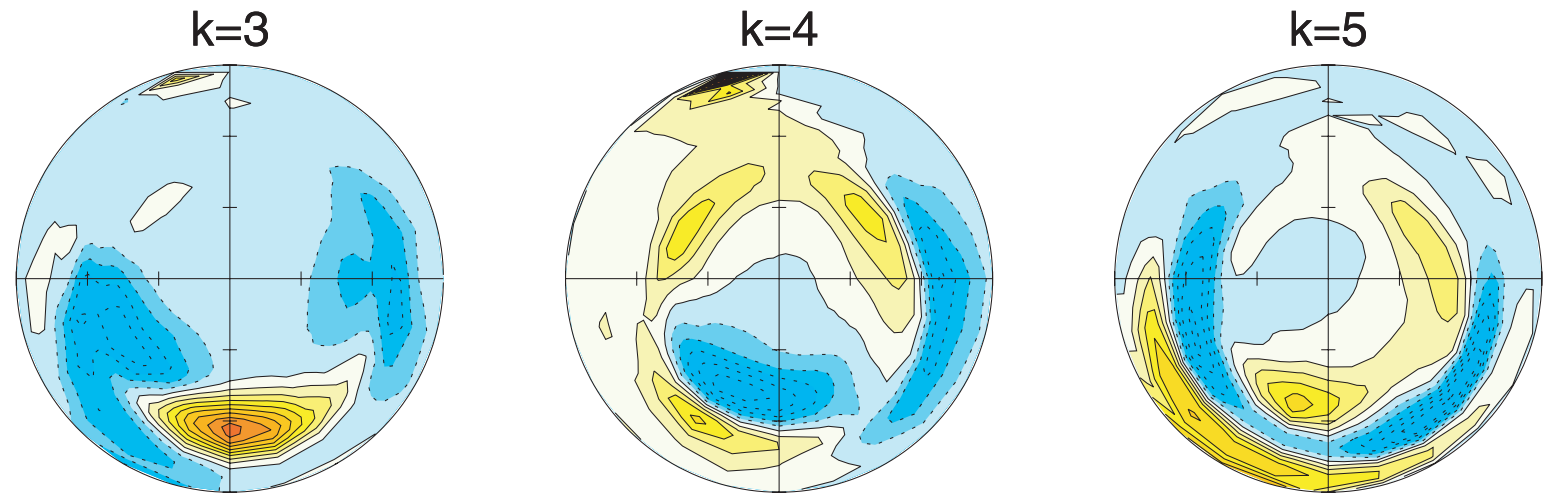

0.1
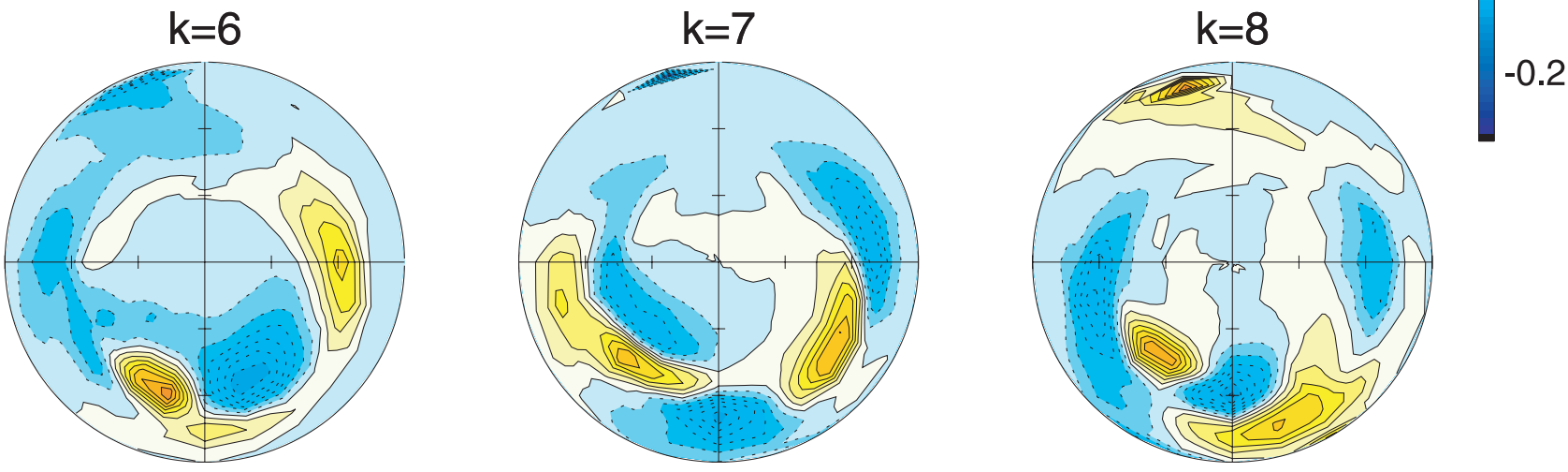

Figure 6. First 9 orthonormal MNOC eigenmodes for UVI images rendered in Apex MLAT-MLT coordinates. Regions of auroral enhancement (quenching) are colored red (blue) according to the normalized color scale at right. 

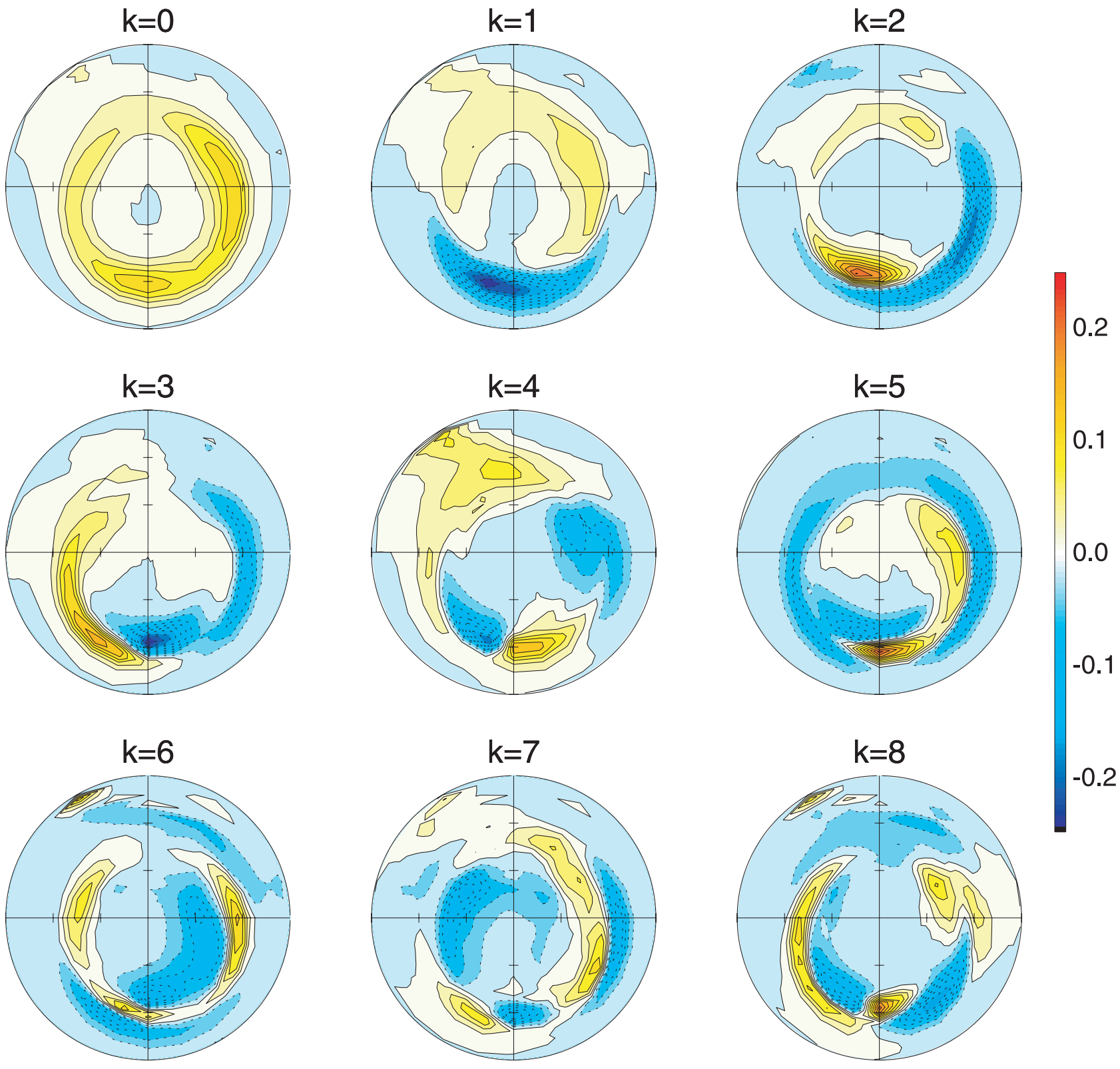

Figure 7. First 9 orthonormal MNOC eigenmodes for UVI images taken during periods for which $\mathrm{AE}<$ $200 \mathrm{nT}$ (in same format as Figure 6). 

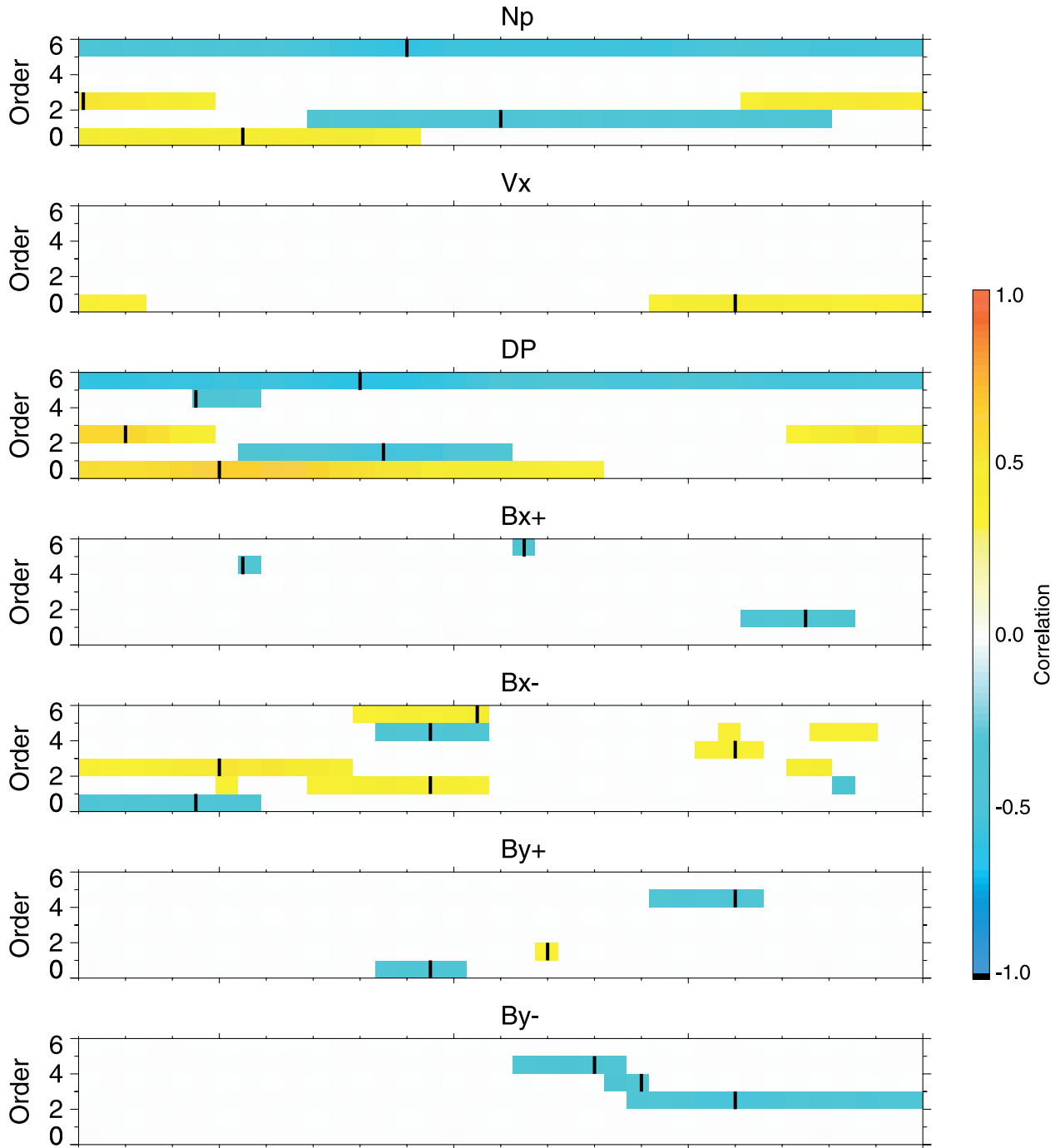

Bz-

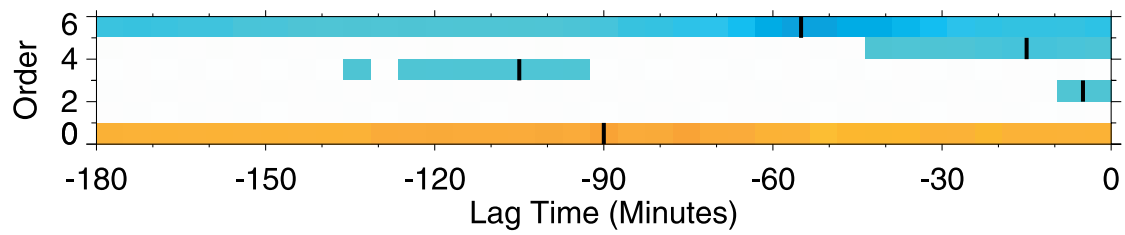

Figure 9. Correlation of MNOC temporal coefficients (vertical axis) against interplanetary parameters during southward IMF with a variable time lag (horizontal axis). Positive (negative) correlations are colored red (blue) according to the scale at right. 

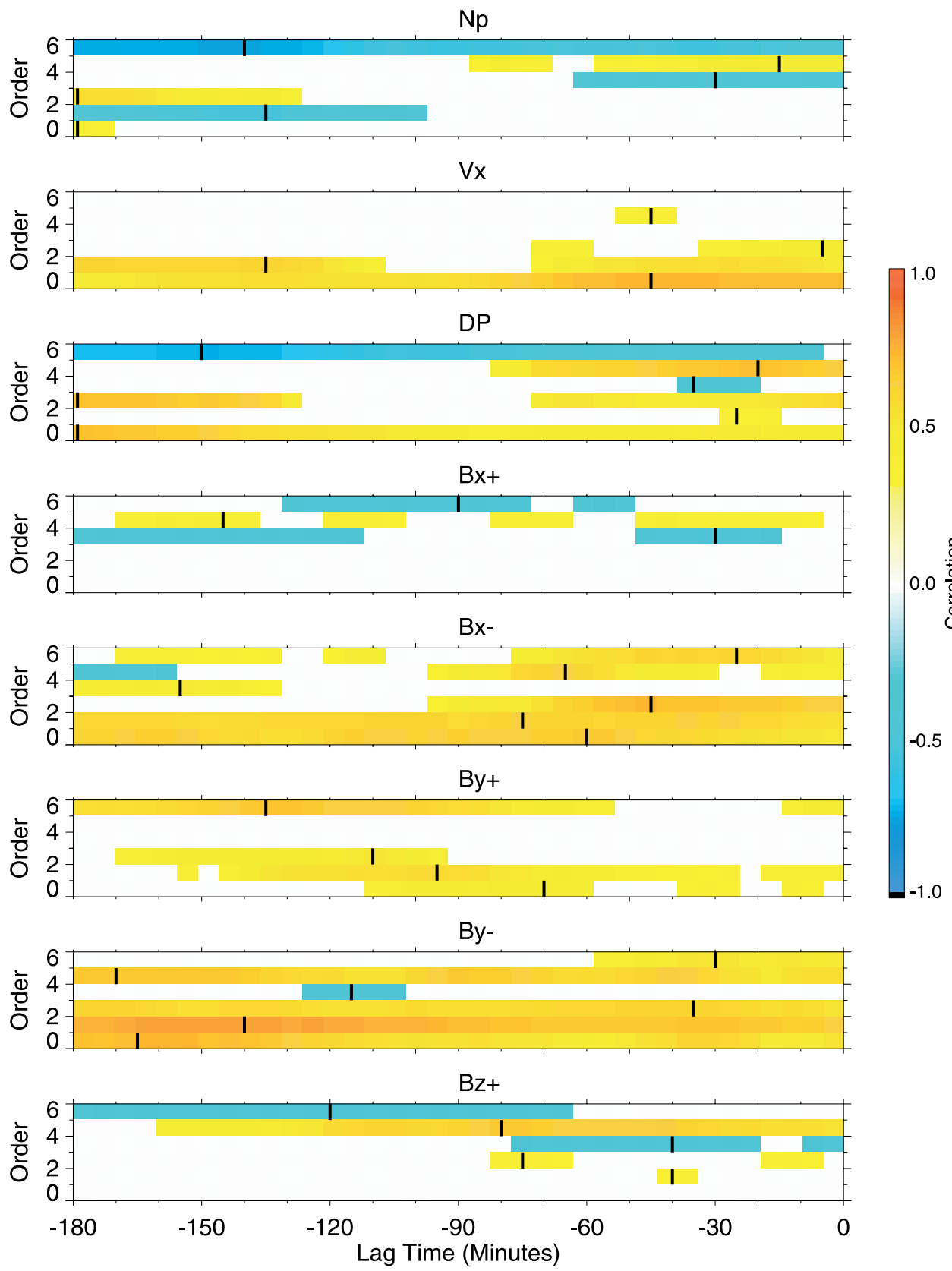

Figure 10. Correlation of MNOC temporal coefficients against interplanetary parameters during northward IMF (in same format as Figure 9). 